\title{
Data-Driven Hybrid Internal Temperature Estimation Approach for Battery Thermal Management
}

\author{
Kailong Liu (D), ${ }^{1}$ Kang Li, ${ }^{2}$ Qiao Peng, ${ }^{3}$ Yuanjun Guo ${ }^{D},{ }^{4}$ and Li Zhang ${ }^{5}$ \\ ${ }^{1}$ School of Electronics, Electrical Engineering and Computer Science, Queen's University Belfast, Belfast BT9 5AH, UK \\ ${ }^{2}$ School of Electronic and Electric Engineering, University of Leeds, Leeds, LS2 9JT, UK \\ ${ }^{3}$ Queen's Management School, Queen's University Belfast, Belfast, BT9 5EE, UK \\ ${ }^{4}$ Shenzhen Institute of Advanced Technology, Chinese Academy of Sciences, Shenzhen, Guangdong 5108055, China \\ ${ }^{5}$ School of Mechatronic Engineering and Automation, Shanghai University, Shanghai 200072, China \\ Correspondence should be addressed to Yuanjun Guo; yj.guo@siat.ac.cn
}

Received 13 April 2018; Accepted 20 May 2018; Published 5 July 2018

Academic Editor: Wenbo Wang

Copyright (c) 2018 Kailong Liu et al. This is an open access article distributed under the Creative Commons Attribution License, which permits unrestricted use, distribution, and reproduction in any medium, provided the original work is properly cited.

\begin{abstract}
Temperature is a crucial state to guarantee the reliability and safety of a battery during operation. The ability to estimate battery temperature, especially the internal temperature, is of paramount importance to the battery management system for monitoring and thermal control purposes. In this paper, a data-driven approach combining the RBF neural network (NN) and the extended Kalman filter (EKF) is proposed to estimate the internal temperature for lithium-ion battery thermal management. To be specific, the suitable input terms and the number of hidden nodes for the RBF NN are first optimized by a two-stage stepwise identification algorithm (TSIA). Then, the teaching-learning-based optimization (TLBO) algorithm is developed to optimize the centres and widths in every neuron of basis function. After optimizing the RBF NN model, a battery lumped thermal model is adopted as the state function with the EKF to filter out the outliers of the RBF model and reduce the estimation error. This data-driven approach is validated under four different conditions in comparison with the linear NN models. The experimental results demonstrate that the proposed RBF data-driven approach outperforms the other approaches and can be extended to other types of batteries for thermal monitoring and management.
\end{abstract}

\section{Introduction}

Rising crude prices of fossil fuels and worldwide concerns of environmental pollution have resulted in the increasing development of clean energy options and energy storage systems [1]. The lithium-ion (Li-ion) battery has exhibited outstanding behaviours such as fast charge capability, no memory effect, long cycle life, and high energy density in comparison with other types of batteries [2]. These excellent performances promote the Li-ion battery to be widely applied as the power sources and energy storage systems in many areas especially for the electric vehicles (EVs) $[3,4]$. Among the applications of existing EVs, a proper battery thermal management system (BTMS) is a key issue to ensure efficient and safe operations for Li-ion battery use [5]. Some battery states including the state of health $(\mathrm{SOH})[6,7]$ and the state of charge (SOC) $[8,9]$ are key parts for the applications of BTMS. Temperature is also a key indication for BTMS, and it affects battery behaviour in many ways such as round trip efficiency, energy and power capability, cycle life, reliability, and charge acceptance.

Estimating the battery temperature is essential for BTMS since it is substantial for managing battery temperature utilization efficiently, prohibiting the battery from being superheat or overcool $[10,11]$. Besides, there is a significant difference between battery internal temperature and surface temperature in high-power applications (e.g., sometimes larger than $\left.15^{\circ} \mathrm{C}[12]\right)$. Superheated internal structure will trigger thermal runaway of the battery and result in safety problem such as electrolyte leakage, even fires and explosion $[13,14]$. Accurate estimation for the battery internal temperature can not only prevent the battery from being damaged 
and further improve battery cycle life but also allow the BTMS to make reasonable control and monitoring strategies to save energy [15]. Therefore, monitoring the surface temperature of the battery is not adequate enough to ensure the battery safety. Estimating the battery internal temperature is crucial for EVs' wider application.

To date, a number of approaches have been presented to obtain the battery internal temperature. One simple and direct approach is to inject the proper microtemperature sensors into the battery cell and get the internal temperature data based on these embedded sensors [16]. However, this approach would cause huge cost and complexity due to the accessional manufacturing requirements and instrumentation challenges especially for the large battery packs that contain several thousand cells.

Some improved approaches for obtaining the battery temperature are to use the numerical thermal models such as the distributed battery thermal model [17] or lumpedparameter battery thermal model $[18,19]$. Kim et al. [17] presented a model-based method combining a reducedorder thermal model and the dual Kalman filter (DKF) for battery temperature estimation under unknown convective conditions. Lin et al. [18] proposed an electrothermal model combining an equivalent-circuit electrical model and a two-stage thermal model to capture the battery surface and internal temperature. These thermal models depend on the information of the battery thermal behaviours, heat generation properties, and battery temperature boundary conditions. However, these numerical thermal models suffer from drawbacks such as the difficulty to get the required information and model parameters.

According to the electrochemical impedance spectroscopy (EIS) [20], some other methods for estimating the battery temperature have been also researched [21, 22]. Richardson et al. [21] proposed an approach based on the EIS to estimate the internal temperature distribution of a battery by using a radial 1-D model and measured battery surface temperature. And in [22], a thermal model together with the electrical impedance measurement, rather than with the direct battery surface temperature measurement, was also developed to estimate the battery internal and surface temperatures. All these EIS-based temperature estimation approaches can be divided into two parts: frequency design and parameter estimation. The former part means to select a proper frequency range which is thermal sensitive but insensitive to other states including SOH and SOC. The latter part means to use measured impedance based on the selected excitations for battery temperature estimation. It should be noticed that different decisions for these two parts would result in significant differences in the accuracy of battery temperature estimation. Determining the suitable frequency and EIS parameters is also difficult, while the selections of these two parts play important roles in designing EIS.

Establishing data-driven models to estimate the battery states based on the artificial intelligence methods including neural networks (NN) [23-25] and support vector machines (SVM) $[26,27]$ has become another hot research area. These data-driven approaches are free of background knowledge and can be applied to capture the highly nonlinear dynamic behaviours of a battery, which are caused by the complicated internal electrochemical reactions. However, to our knowledge, these data-driven approaches are primarily applied to estimate the battery $\mathrm{SOH}$ or SOC, but very few attempts have been done for the battery temperature estimation, especially for the internal temperature. The battery internal temperature is also a crucial internal state because it affects the safety and behaviours of a battery directly. Our early research [28] proposed a linear NN model-based approach to estimate Li-ion battery internal temperature. Although the internal temperature has been successfully estimated by training the $\mathrm{NN}$ model, the results are still less satisfactory in some temperature conditions due to the poor generalization ability of the linear NN model.

In this paper, a hybrid RBF NN-based approach together with the EKF is presented to estimate the battery internal temperature. The battery dynamics is a complicated process characterized by nonlinearity, uncertainty, and multivariable coupling, and the RBF NN model is used to capture and describe the dynamics of the battery. Elements including terminal voltage, battery current, and surface temperature are selected as the RBF inputs, while the internal temperature of the battery is selected as the output of the RBF NN model. In order to remove the redundant terms which may lead to overfitting, a TSIA [29] is first developed to determine the most significant input terms. And the number of the hidden layer neurons of RBF is also determined by the FRA [30] in the forward selection stage of the TSIA, where the structure of the RBF NN model is optimized. In order to get the optimized parameters in the radial kernel function, a newly developed meta-heuristic algorithm named TLBO [31] is then developed to optimize the centres and widths in every neuron of basis function. After that, a battery lumped thermal model is adopted as the state function with the EKF to filter out the outliers in the RBF NN estimation, aimed at reducing the estimation errors of the RBF NN model.

The remainder of this paper is organized as follows. The detailed preliminaries of the RBF NN, the linear NN, the TSIA, the TLBO procedure, and the battery lumped thermal model are presented in Section 2. Section 3 illustrates the complete TSIA-based RBF NN procedure for estimating the battery internal temperature, followed by the EKF to reduce the estimation errors. Section 4 gives the estimation results of our hybrid approach, and the effectiveness of the proposed RBF NN EKF-based approach is analysed in comparison with the solo RBF NN approach, the solo linear NN approach, and the linear NN EKF-based approach. Finally, Section 5 concludes the paper.

\section{Preliminaries}

This section presents the preliminaries of the proposed method, including the RBF NN, the TSIA, the TLBO procedure, and the battery lumped thermal model. In order to analyse the effectiveness of the hybrid approach with other types of the NN model, the linear NN is also reviewed briefly in this section. 


\subsection{Principle of the Neural Network}

2.1.1. Radial Basis Function of the Neural Network. The neural network is the artificial intelligence technology which has been widely adopted in the system modelling area [32]. The RBF NN is a forward network model with good generalization capacity [33]. It has a strong approximation ability to identify the nonlinear system with the simple structure and is widely used as a strong computational tool in the state estimation area. For the RBF NN, the activated function of the hidden layer neurons is the radial basis function, and every neuron has two key parameters to describe the function's centre and its width, respectively. The structure of the typical RBF NN is illustrated in Figure 1. The Gaussian function is the former of the $i$ th $\mathrm{RBF}$ hidden neuron illustrated as follows:

$$
\varphi_{i}(X)=\exp \left[-\frac{\left(X-c_{i}\right)^{2}}{2 \sigma_{i}^{2}}\right], \quad \text { for } i=1,2, \ldots, n
$$

where $c_{i}$ and $\sigma_{i}$ are the centre and width of the $i$ th hidden neuron, respectively, in the hidden layer. Thus, a general multi-input and single-output (MISO) RBF NN can be formulated as

$$
y(t)=\sum_{i=1}^{n} \omega_{i} \cdot \varphi_{i}\left(\mathbf{X}(t), c_{i}, \sigma_{i}\right)+e(t),
$$

where $y(t)$ and $\mathbf{X}(t)$ stand for the model output and input vector at time instant $t$, respectively. $\omega_{i}$ is the output weight for the $i$ th hidden neuron with a total number of $n$ neurons. $e(t)$ stands for the RBF modelling error. A set of $j$ samples is applied for training the RBF model; then, the output is shown as

$$
\widehat{Y}=\Phi \cdot W
$$

where $\widehat{Y}=\left[\widehat{y}_{1}, \hat{y}_{2}, \ldots, \hat{y}_{n}\right]^{T}, \Phi=\left[\varphi_{1}, \varphi_{2}, \ldots, \varphi_{n}\right]^{T}$, and $W=$ $\left[\omega_{1}, \omega_{2}, \ldots, \omega_{n}\right]$. Defining the actual system output as $Y=$ $\left[y_{1}, y_{2}, \ldots, y_{n}\right]$, then the RBF modelling error function can be given as

$$
e=Y-\widehat{Y}=Y-\Phi \cdot W
$$

In order to minimize the error function in (4) after fixing the centres and widths in the RBF $\mathrm{NN}$, the least square method can be applied to determine the linear output weights $W$ as

$$
W=\left(\Phi^{T} \Phi\right)^{-1} \Phi^{T} \cdot Y
$$

So for constructing the RBF NN model, one of the main challenges is the input selection. For system modelling,

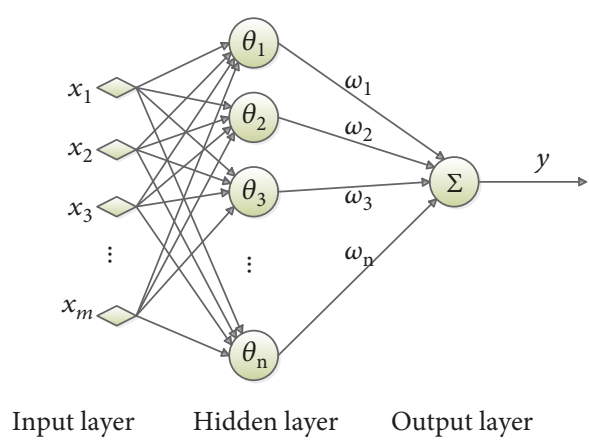

FIgURE 1: Structure of a MISO RBF NN.

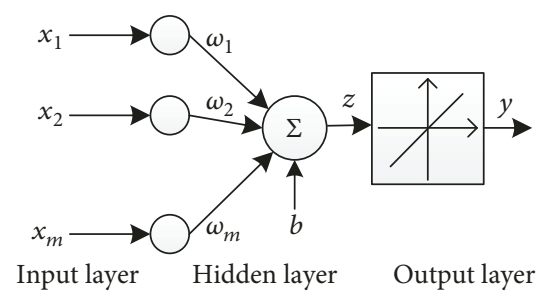

FIGURE 2: Structure of a linear NN.

some data sets would be irrelevant or have little effect on the output; thus, the inclusion of them may result in overfitting problem and high computational cost [34]. Removing these redundant input terms is essential to improve the accuracy and generalization capacity of the NN model. On the other side, the number of neurons in the hidden layer also plays important role in the accuracy of the NN model. Too many neurons will lead to a risk of overfitting and bad generalization capacity, while too few neurons will also cause the underfitting problem. Determining the number of hidden neurons which can be adequate enough to cover the space of the input vector is also a difficult problem. Some approaches can be adopted to determine the optimal input terms for the RBF NN and quantify the number of hidden neurons, but there is no general solution. The TSIA is applied to select the proper input terms and determine the number of the hidden layer neurons of RBF in this study. The principles of the TSIA and the corresponding optimizing procedure are described in Sections 2.2 and 3.1, respectively.

2.1.2. Linear Neural Network. In order to demonstrate the effectiveness of the proposed hybrid approach using the $\mathrm{RBF}$ NN, a linear NN is also adopted and compared. The typical structure of the linear NN with $m$ dimension inputs and one output is shown in Figure 2. It consists of a mass of simple neurons. The main elements of the linear NN can be divided into five parts: (1) the input vector $\mathbf{X}=$ $\left[x_{1}, x_{2}, x_{3}, \ldots, x_{m}\right]^{T},(2)$ the weights between the input and the hidden layer $w_{1}, w_{2}, w_{3}, \ldots, w_{m}$, (3) the summation unit $Z$, (4) the continuously differentiable transfer function $f(x)$, and (5) the output $y$. 
As shown in Figure 2, $z=\sum_{i=1}^{m} x_{i} \omega_{i}+b$ and $y=$ $f\left(\sum_{i=1}^{m} x_{i} \omega_{i}+b\right)$. In this study, the transfer function in the linear NN model is selected as $f(x)=x$ for the battery internal temperature estimation.

2.2. Principle of a Two-Stage Stepwise Identification Algorithm. The TSIA is a method to both optimize the model structure and estimate the model parameters [29]. In this study, (2) is approximated with a polynomial nonlinearautoregressive-exogenous (NARX) model, and the input selection of the NN model can be transformed to a procedure of model term selection; then, the TSIA is adopted to choose the most significant input terms. Besides, the optimal number of hidden layer neurons is also determined by the forward selection step named FRA in the TSIA.

The TSIA for input selection can be divided into a forward selection step and a second refinement step. The forward selection is similar to a FRA researched in [30]. For this step, a recursive matrix $\mathbf{M}_{k}$ and a residual matrix $\mathbf{R}_{k}$ are first introduced and defined as

$$
\begin{aligned}
& \mathbf{M}_{k}=P_{k}^{T} P_{k}, \quad k=1, \ldots, n, \\
& \mathbf{R}_{k}=I-P_{k} M_{k}^{-1} P_{k}^{T}, \quad R_{0}=I,
\end{aligned}
$$

where $P_{k}=\left[p_{1}, p_{2} \ldots, p_{k}\right]$ includes the first $k$ selected terms of the full regression matrix. $\mathbf{R}_{k}$ is the matrix term with the following attractive properties [30]:

$$
\begin{aligned}
R_{k}^{T} & =R_{k}, \\
\left(R_{k}\right)^{2} & =R_{k}, \\
R_{k} R_{j} & =R_{j} R_{k}=R_{k}, \quad \text { for all } k \geq j, \\
R_{k+1} & =R_{k}-\frac{R_{k} p_{k+1} p_{k+1}^{T} R_{k}^{T}}{p_{k+1}^{T} R_{k} p_{k+1}}, \quad k=0,1, \ldots, n-1, \\
R_{1, \ldots, p, \ldots, q, \ldots, k} & =R_{1, \ldots, q, \ldots, \ldots, \ldots, k}, \quad p, q \leq k .
\end{aligned}
$$

According to (10), any changes in the selection order of the regressor terms $p_{1}, p_{2}, \ldots, p_{k}$ would make no difference to the residual matrix $\mathbf{R}_{k}$. This property is able to decrease the computation cost for the second refinement step. Suppose $E_{k}$ means the square error after determining $k$ terms which is also the cost function for the forward selection step, and it can be defined as

$$
E_{k}=y^{T} R_{k} y
$$

According to (11), the cost function decreases after adding new $k+1$ th term. The net contribution of $p_{k+1}$ will be calculated as

$$
\Delta E_{k+1}=y^{T}\left(R_{k}-R_{k+1}\right) y=\frac{y^{T} R_{k} p_{k+1} p_{k+1}^{T} R_{k} y}{p_{k+1}^{T} R_{k} p_{k+1}}=\frac{\left(y^{T} p_{k+1}^{(k)}\right)^{2}}{p_{k+1}^{T} p_{k+1}^{(k)}} .
$$

To further simplify calculation and reduce the computational cost, two quantities are denoted as

$$
\begin{aligned}
a_{k, i} & =\left(p_{k}^{(k-1)}\right)^{T} p_{i}^{(k-1)}, \\
a_{1, i} & =p_{1}^{T} p_{i}, \\
i & =k, \ldots, n, \\
b_{k} & =\left(p_{k}^{(k-1)}\right)^{T} y, \quad k=1,2, \ldots, n, \\
b_{1} & =\left(p_{1}^{(0)}\right)^{T} \quad y=p_{1}^{T} y .
\end{aligned}
$$

We can define an auxiliary matrix $\mathbf{A} \in R^{n \times n}$ and a vector $\mathbf{b} \in R^{n \times 1}$ with elements given by

$$
\begin{gathered}
a_{k, i}=p_{k}^{T} p_{i}-\frac{\sum_{l=1}^{k-1} a_{l, k} a_{l, i}}{a_{l, l}}, \\
b_{k}=p_{k}^{T} y-\frac{\sum_{l=1}^{k-1} a_{l, k} b_{l}}{a_{l, l}} .
\end{gathered}
$$

Then, the net contribution of an added term to decrease the cost function value is denoted as

$$
\Delta E_{k+1}=\frac{b_{k+1}^{2}}{a_{k+1, k+1}} .
$$

By this method, the terms from the candidate pool will be chosen continuously for which term makes the largest contribution in the forward selection stage. The sum squared error (SSE) is selected as the terminal criterion for the selection process, which means that the forward selection process would stop when the total net contribution of the selected terms is larger than a predetermined value.

After that, the procedure will move to the second refinement step, which is aimed at eliminating the insignificant input selection terms caused by the constraint in forward construction.

Suppose a selected item $p_{k}$ is shifted to the $n$th position in $P_{k}$. Two adjacent terms will be interchanged repeatedly as

$$
\begin{aligned}
p_{q}^{*} & =p_{q+1}, \\
p_{q+1}^{*} & =p_{q}, \\
q & =k, \ldots, n-1,
\end{aligned}
$$

where $*$ indicates the updated value. Then, the $R_{q}$ will be updated using

$$
R_{q}^{*}=R_{q-1}-\frac{R_{q-1} p_{q}^{*}\left(p_{q}^{*}\right)^{T} R_{q-1}^{T}}{\left(p_{q}^{*}\right)^{T} R_{q-1} p_{q}^{*}} .
$$


The following terms will be also updated at the same time:

For the matrix $\mathbf{A}$, the $q$ th and the $(q+1)$ th columns with the elements from row 1 to $q-1$ will be modified by

$$
\begin{aligned}
a_{i, q}^{*} & =a_{i, q+1}, \\
a_{i, q+1}^{*} & =a_{i, q}, \\
i & =1, \ldots, q-1 .
\end{aligned}
$$

The $q$ th row with elements from column $q$ to column $n$ is also modified according to

$$
a_{q, j}^{*}=\left\{\begin{array}{l}
\frac{a_{q+1, q+1}+a_{q, q+1}^{2}}{a_{q, q}}, \quad j=q, \\
a_{q, q+1}, \quad j=q+1, \\
\frac{a_{q+1, j}+a_{q, q+1} a_{q, j}}{a_{q, q}}, \quad j \geq q+2 .
\end{array}\right.
$$

And the $(q+1)$ th row $a_{q+1, j}$ for $j=q+1, \ldots, n$ is also modified according to

$$
a_{q+1, j}^{*}= \begin{cases}\frac{a_{q, q}-a_{q, q+1}^{2}}{a_{q, q}^{*}}, & j=q+1, \\ \frac{a_{q, j}-a_{q, q+1} a_{q, j}^{*}}{a_{q, q}^{*}}, & j \geq q+2 .\end{cases}
$$

For the vector $\mathbf{b}$, the $q$ th and the $(q+1)$ th elements will be only changed by

$$
\begin{array}{r}
b_{q}^{*}=\frac{b_{q+1}+a_{q, q+1} b_{q}}{a_{q, q}}, \\
b_{q+1}^{*}=\frac{b_{q}-a_{q, q+1} b_{q}^{*}}{a_{q, q}^{*}} .
\end{array}
$$

This updated process will continue until the $k$ th term is set to the $n$th position; then, the shifted new regression matrix and the residue matrix series become

$$
\begin{aligned}
& \mathbf{P}_{n}^{*}=\left[p_{1}, \ldots, p_{k-1}, p_{k+1}, \ldots, p_{n}, p_{k}\right], \\
& \mathbf{R}_{k}^{*}=\left[R_{1}, \ldots, R_{k-1}, R_{k}^{*}, \ldots, R_{n}^{*}\right] .
\end{aligned}
$$

As the term has been moved to the $n$th position in $P_{k}$, contributions of the moved term will be reviewed by (15). The moving and comparing process is repeated until all of the selected terms become more significant than those remaining in the candidate term pool. Finally, the satisfactory terms will be chosen to constitute the optimal $\mathrm{NN}$ inputs.

2.3. Principle of Teaching-Learning-Based Optimization. The TLBO algorithm is a population-based method that mimics the nature of a typical teaching and learning procedure in a class $[35,36]$. The optimization procedure can be divided into two parts, namely, the teaching stage and learning stage. For the teaching stage, a teacher will be first selected in every learning generation and improve the knowledge of students.
Then, for the learning stage, students will gain potential useful information from a mutual interaction with counterparts.

The conventional training process for the RBF NN applies the stochastic gradient method to adjust the nonlinear parameters including centres and widths in the $\mathrm{RBF}$ NN. The primary drawback by using the stochastic gradient method is that the training method is easily prone to being trapped in a local minimum. The TLBO is applied to train the RBF NN by optimizing the RBF centre $c_{i}$ and width $\sigma_{i}$ in this study. It is convenient and easy to adopt TLBO for the parameter optimization of the RBF NN since there is no algorithm-specific parameter that needs to be adjusted by a user during algorithm implementation. The typical framework of TLBO is shown in Figure 3.

2.4. Principle of a Battery Lumped Thermal Model. In order to use the EKF to filter out the outliers of the estimation results, a proper battery model which is capable of describing the battery thermal behaviour needs to be selected as the state function. Because the simple structure and parameters are easy to be identified, a battery lumped thermal model is adopted with the EKF to filter out outliers in this study.

Assuming that the battery surface and internal temperatures are both uniform, the battery has a uniform distribution of heat generation. Heat conduction is the only heat transfer form between the ambience and the battery surface and also between the battery interior and the surface, and a two-stage battery cell thermal model is then described as $[37,38]$

$$
\begin{aligned}
& \frac{C_{1} \cdot d T_{\text {in }}}{d t}=k_{1} \cdot\left(T_{\mathrm{sh}}-T_{\mathrm{in}}\right)+I^{2} \cdot R, \\
& \frac{C_{2} \cdot d T_{\mathrm{sh}}}{d t}=k_{1} \cdot\left(T_{\mathrm{in}}-T_{\mathrm{sh}}\right)+k_{2} \cdot\left(T_{\mathrm{amb}}-T_{\mathrm{sh}}\right),
\end{aligned}
$$

where $I$ stands for the battery current. $T_{\text {in }}$ and $T_{\text {sh }}$ are the battery interior temperature and shell temperature, respectively. $T_{\mathrm{amb}}$ is the ambient temperature of the battery. $R$ is the internal resistance of the battery. Both $C_{1}$ and $C_{2}$ stand for the heat capacities. $k_{1}$ is a thermal dissipation rate to represent the heat conduction happening between the battery interior and surface, while $k_{2}$ is another thermal dissipation rate to stand for the heat conduction between the ambience and battery surface [39].

Supposing $d T(k+1) / d t=(T(k+1)-T(k)) / T_{s}$, the battery lumped thermal model is then further denoted as

$$
\begin{aligned}
& T_{\mathrm{in}}(k+1)=A_{1} \times T_{\mathrm{in}}(k)+A_{2} \times T_{\mathrm{sh}}(k)+A_{3} \times I^{2}(k), \\
& T_{\mathrm{sh}}(k+1)=B_{1} \times T_{\mathrm{in}}(k)+B_{2} \times T_{\mathrm{sh}}(k)+B_{3} \times T_{\mathrm{amb}},
\end{aligned}
$$

where $T_{\mathrm{s}}$ stands for the sampling time period. $A_{1}=(1-$ $\left.T_{\mathrm{s}} \cdot k_{1}\right) / C_{1}, A_{2}=\left(T_{\mathrm{s}} \cdot k_{1}\right) / C_{1}, A_{3}=\left(T_{\mathrm{s}} \cdot R\right) / C_{1}, B_{1}=\left(T_{\mathrm{s}} \cdot k_{1}\right) /$ $C_{2}, B_{2}=\left(1-T_{s} \cdot\left(k_{1}+k_{2}\right)\right) / C_{2}$, and $B_{3}=\left(T_{s} \cdot k_{2}\right) / C_{2}$.

\section{RBF NN Model-Based Internal Temperature Estimation}

The RBF NN model is first trained and optimized by the TSIA and TLBO, respectively. Then, the well-designed RBF 


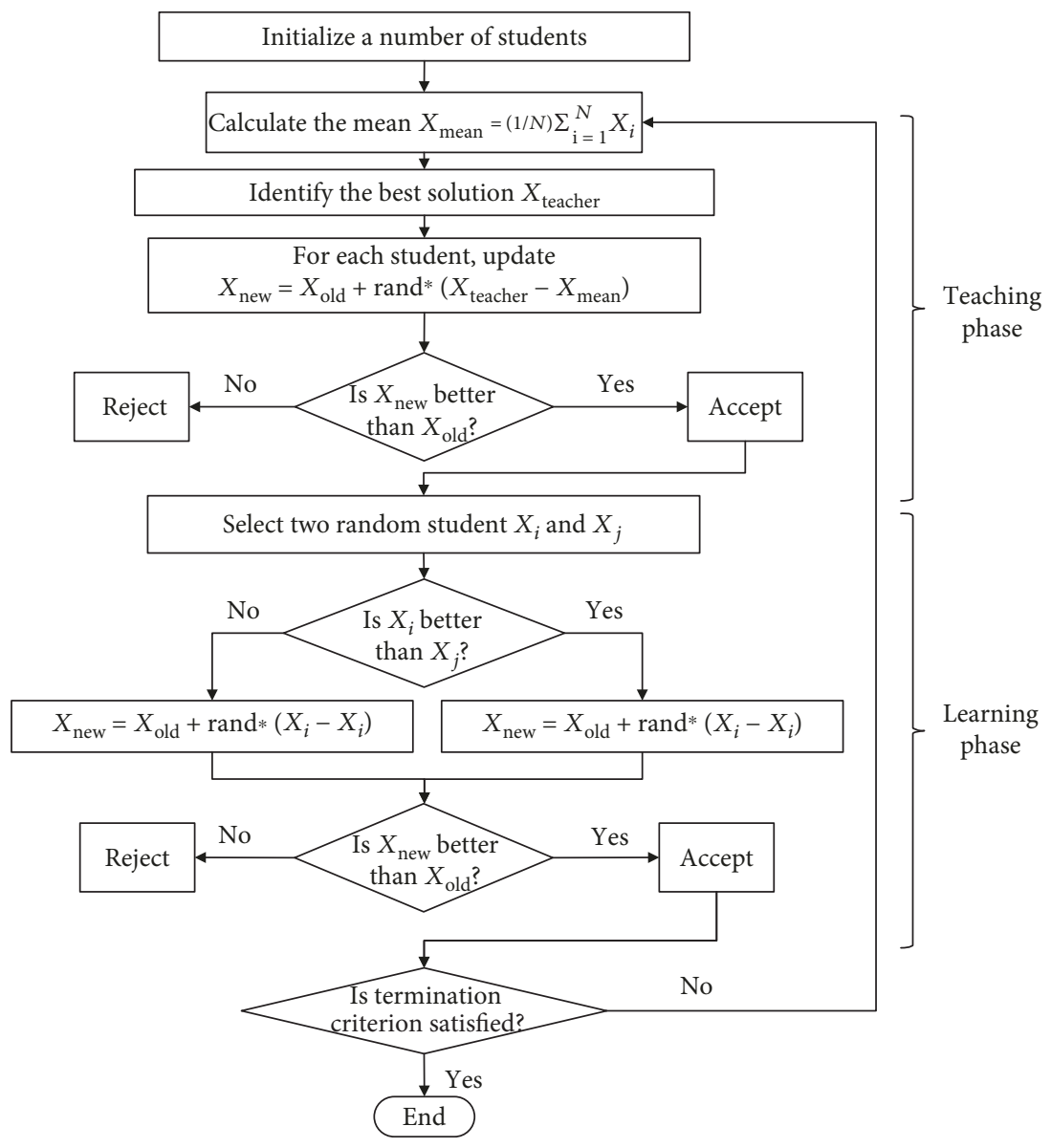

Figure 3: Typical framework of the TLBO.

NN model is applied for the internal temperature estimation of the Li-ion battery. The terminal voltage $V$, battery current $I$, and surface temperature $T_{\text {sh }}$ are selected as the elements of the RBF NN inputs, and the battery internal temperature $T_{\text {in }}$ is chosen as the only one neuron of the NN output layer. On the one hand, $T_{\text {sh }}$ is an important index to describe the battery thermal behaviour and has the direct impacts on $T_{\text {in }}$. On the other hand, there is a nonlinear relationship between the $V$ and $T_{\text {in }}$. Besides, the battery current $I$ will result in the battery heat generation and further affects $T_{\text {in }}$. Therefore, three essential factors including $T_{\text {sh }}, V$, and $I$ are chosen as the input elements for the RBF NN model to estimate $T_{\text {in }}$.

The overall flow diagram for the TSIA-based RBF NN internal temperature estimation approach is illustrated in Figure 4 . The proposed hybrid $T_{\text {in }}$ estimation approach is described summarily in the following steps:

Step 1. Collect the battery operating data by the battery operation platform. The data includes the battery terminal voltage, current, surface temperature, and internal temperature.

Step 2. Normalize all of the battery operating data.

Step 3. Prepare the data candidate pool for the RBF NN training. The data for the $V, I$, and $T_{\text {sh }}$ are selected as the candidate inputs, and the $T_{\text {in }}$ is the output for the RBF training.

Step 4. Use the TSIA to determine the significant input terms for RBF NN and the FRA in the first stage of the TSIA to optimize the numbers of hidden layer neurons of RBF; then, optimize the structure of the RBF NN.

Step 5. Use the TLBO to optimize the centres and widths in the RBF NN. The corresponding weights between RBF hidden and output layers are calculated by the least square approach.

Step 6. When the optimizing procedure is finished, save the centres, widths, and the corresponding weights between hidden and output layers in the RBF NN model.

Step 7. Use the trained RBF NN model for the battery $T_{\text {in }}$ estimation. An identified lumped thermal model is combined with the EKF to filter out the outliers and improve the model estimation accuracy.

3.1. Structure Optimization of the RBF NN Model. In this study, the TSIA approach is used firstly by calculating the net contribution of every added input term and then the most 


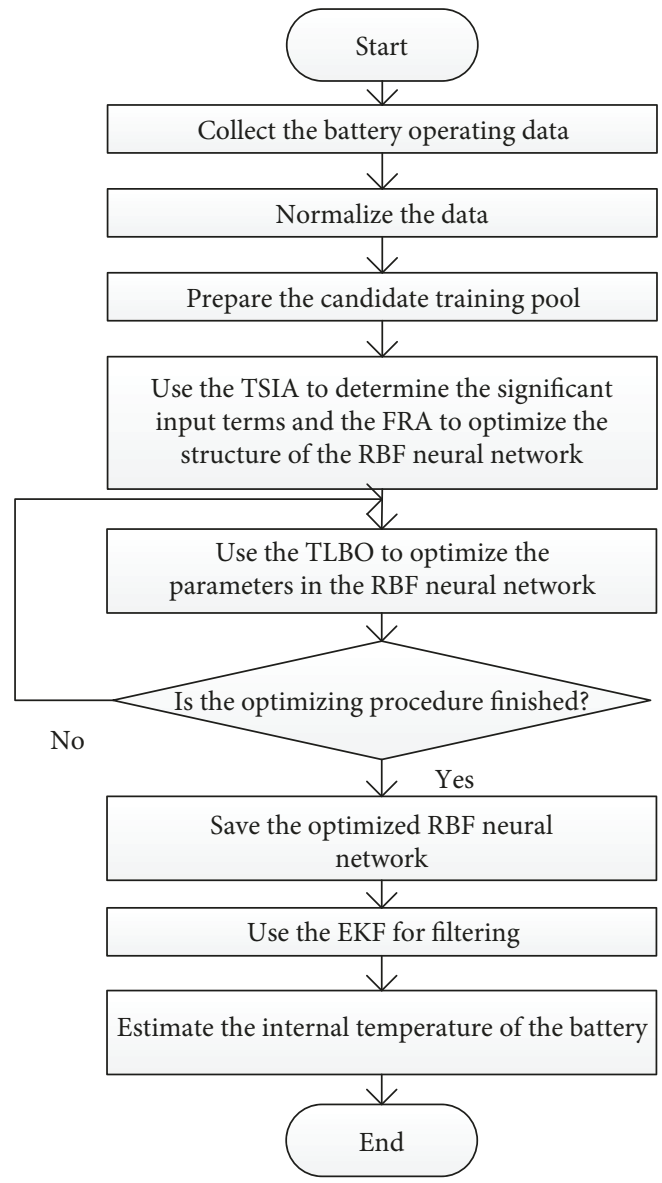

FIGURE 4: The overall flow diagram of the proposed TSIA-based RBF NN internal temperature estimation.

significant inputs are selected. After that, the forward selection stage which is also named FRA in the TSIA method is applied to determine the proper number of hidden layer neurons of the RBF NN; then, the structure of the RBF NN can be optimized.

The detailed procedure to determine the construction of the RBF NN for estimating the battery $T_{\text {in }}$ by using the TSIA method is shown as follows.

(1) Select the significant terms for the RBF NN input.

(a) Formulate the candidate input term pool that consists of $T_{\mathrm{sh}}, V, I$, and their delays $T_{\mathrm{sh}}\left(t-k_{\mathrm{sh}}\right)$, $V\left(t-k_{v}\right)$, and $I\left(t-k_{i}\right) .\left(k_{\mathrm{sh}}, k_{v}\right.$, and $k_{i}$ are the delays of the surface temperature, terminal voltage, and battery current, resp.)

(b) For the forward selection stage, compute the input term net contributions according to (15) and select the largest contributors until the terminal criterion SSE is achieved.

(c) For the second refinement stage, move and compare the selected terms to eliminate the insignificant input selection terms. These processes are repeated until no more reduction can be achieved.
(2) Determine the optimal number of hidden neurons.

After choosing the satisfactory input terms for the RBF model, the contributions of the hidden neurons need to be calculated. The selected input terms will form a new term pool. And the SSE can provide an effective criterion to determine the RBF hidden neurons by using the FRA. This process will continue until the expected total net contribution value of the added hidden neurons is achieved. Then, the number of the hidden layer neurons is optimized as well as the construction of the RBF NN.

3.2. Parameter Optimization of the RBF NN Model. After determining the construction of the RBF NN model by the TSIA method, the nonlinear parameters including $c_{i}$ and $\sigma_{i}$ in the radial kernel function need to be optimized by the TLBO algorithm. The root mean squared error (RMSE) is selected as the fitness function to be minimized, and it can be expressed as follows:

$$
\min f=\operatorname{RMSE}=\sqrt{\frac{1}{N} \cdot \sum_{i=1}^{N}\left(\hat{y}-y_{m}\right)},
$$

where $\hat{y}$ is the value by estimation and $y_{m}$ stands for the actual data by measurement.

The initial particles of TLBO are obtained by randomly assigning values for $c_{i}$ and $\sigma_{i}$. A better set of nonlinear parameters is determined through the TLBO process; then, a more accurate model with lower RMSE will be obtained. The detailed optimization procedure for RBF nonlinear parameters by TLBO is described as follows:

(1) TLBO initialization:

(a) Set the numbers of generations $G_{m}$, population sizes $N_{p}$, maximum and minimum bounds of the solutions $\mathrm{St}_{\max }$ and $\mathrm{St}_{\min }$.

(b) Randomly generate the particles in which each hidden neuron can contain two unknowns $c_{i}$ and $\sigma_{i}$.

(2) Teaching stage:

(a) The values of the fitness function $f$ for all solutions are computed to determine the best result as the teacher $X_{\text {teacher }}$.

(b) The mean $X_{\text {mean }}$ of the population is calculated column-wise.

(c) The teacher will try to move the mean from $X_{\text {mean }}$ to $X_{\text {teacher }}$. Then, the difference $\mathrm{DM}_{t}$ between $X_{\text {mean }}$ and $X_{\text {teacher }}$ is calculated.

(d) The obtained difference $\mathrm{DM}_{t}$ is added to the current solution to improve the knowledge of learners.

(e) The better solution in learners after the effect of the teacher is then accepted. 
(3) Learning stage:

(a) Students share knowledge to each other and obtain improvement through the interactions.

(b) Select better solutions after the interaction of students.

(c) Go back to the teaching stage until the final generation is reached or the defined criterion is met.

3.3. Application of the EKF to Improve Accuracy. After determining the construction and the corresponding parameters, the well-trained NN model is applied to estimate the battery $T_{\text {in }}$. An EKF is also used to filter out the outliers of the NN model output, aimed at reducing the errors and at improving the estimation accuracy.

The summary process of the EKF is depicted in Table 1.

The battery $T_{\text {in }}$ outputs from the NN model are determined as the noisy measurement to develop a normal battery state-space model for the estimation of the EKF. Therefore, the state function is the battery lumped thermal model. And the measurement function consists of the welltrained NN model. The target of the EKF is to filter out the outliers in the NN output and decrease the estimation errors [40]. The state-space model for the EKF is described as follows:

State function:

$$
\left[\begin{array}{c}
T_{\mathrm{in}}(k+1) \\
T_{\mathrm{sh}}(k+1)
\end{array}\right]=\left[\begin{array}{cc}
A_{1} & A_{2} \\
B_{1} & B_{2}
\end{array}\right]\left[\begin{array}{c}
T_{\mathrm{in}}(k) \\
T_{\mathrm{sh}}(k)
\end{array}\right]+\left[\begin{array}{c}
A_{3} \times I^{2}(k) \\
B_{3} \times T_{\mathrm{amb}}
\end{array}\right]+v
$$

Measurement function:

$$
\left[\begin{array}{c}
\mathrm{NN}(k) \\
T_{\mathrm{sh}}(k)
\end{array}\right]=\left[\begin{array}{c}
T_{\mathrm{in}}(k) \\
T_{\mathrm{sh}}(k)
\end{array}\right]+\omega,
$$

where $\mathrm{NN}(k)$ stands for the $T_{\text {in }}$ estimated by the NN model at an instant period $k . v$ is the state noise, while $\omega$ is the measurement noise.

The developed approach which consists of the welltrained NN model and EKF for battery $T_{\text {in }}$ estimation is shown in Figure 5.

\section{Experiments and Results}

4.1. Battery Data and Platform. The experiment data of the Li-ion battery were tested from our EV lab in Queen's University Belfast. These data are applied for the training and validation of the NN model. The operation platform of the battery consists of a charger to charge the battery, an electric load to discharge the battery, and a thermal cabinet to control the testing environment, as shown in Figure 6 [41]. The battery applied for testing in our lab is a prismatic $\mathrm{LiFePO}_{4}$-graphite battery. The nominal capacity and the operation voltage of the battery are $10 \mathrm{Ah}$ and $3.2 \mathrm{~V}$, respectively. Some charging and discharging tests under different
TABLE 1: Summary process of the EKF.

State-space model:

$$
\begin{aligned}
& s_{k+1}=f\left(s_{k}, u_{k}\right)+w_{k}, \\
& y_{k}=g\left(s_{k}, u_{k}\right)+v_{k},
\end{aligned}
$$

where $w_{k}$ and $v_{k}$ are independent, zero-mean Gaussian noise processes.

Definitions:

$$
\begin{aligned}
& A_{k}=\left.\left(\partial f\left(s_{k}, u_{k}\right) / \partial s\right)\right|_{s_{k}=\widehat{s}_{k}^{+}}, \\
& C_{k}=\left.\left(\partial g\left(s_{k}, u_{k}\right) / \partial s\right)\right|_{s_{k}=\widehat{s_{k}}} .
\end{aligned}
$$

Initialization: for $k=0$,

Set $\widehat{s}_{0}^{+}=E\left[s_{0}\right]$,

$$
\sum_{\tilde{s}, 0}^{+}=E\left[\left(s_{0}-E\left[s_{0}\right]\right)\left(s_{0}-E\left[s_{0}\right]\right)^{T}\right] .
$$

Computation: for $k=1,2, \ldots$, compute

Time update:

Measurement update:

$$
\begin{aligned}
& \widehat{s_{k}}=f\left(\widehat{s}_{k-1}^{+}, u_{k-1}\right), \\
& \sum_{\tilde{s}, k}^{-}=A_{k-1} \sum_{\tilde{s}, k-1}^{+} A_{k-1}^{T}+\sum w .
\end{aligned}
$$

$$
\begin{aligned}
& L_{k}=\sum_{\tilde{s}, k}^{-} C_{k}^{T}\left[C_{k} \sum_{\tilde{s}, k}^{-} C_{k}^{T}+\sum v\right]^{-1}, \\
& \widehat{s}_{k}^{+}=\widehat{s_{k}}+L_{k}\left[y_{k}-g\left(\widehat{s_{k}}, u_{k}\right)\right], \\
& \sum_{\tilde{s}, k}^{+}=\left(I-L_{k} C_{k}\right) \sum_{\tilde{s}, k}^{-} .
\end{aligned}
$$

current levels are operated to obtain the corresponding data including $I, V, T_{\mathrm{sh}}$, and $T_{\mathrm{in}} . T_{\mathrm{amb}}$ is set as $24^{\circ} \mathrm{C}$ by the thermal cabinet for these tests.

According to the proper normalization of data, the training procedure of the NN model will be more efficient with higher robustness. Therefore, before training the NN model, the data will be normalized by

$$
x=\frac{2\left(x-x_{\min }\right)}{\left(x_{\max }-x_{\min }\right)-1},
$$

where $x_{\max }$ and $x_{\min }$ are the upper and lower limits for the data vector $\mathbf{x}$ of the NN. When validating the model accuracy, the testing data will be also scaled by the same $x_{\max }$ and $x_{\min }$ adopted in the training step.

4.2. Identification of the Battery Lumped Thermal Model. Before filtering out the outliers and decreasing the output noises in the NN model by the EKF algorithm, the battery lumped thermal model (state function) should be first identified based on the experimental data. The self-heating experimental data are adopted for battery thermal model identification. The constant parameters are identified by the least square method. Details about the corresponding identification procedure can be found in our previous work [41] and will not be presented due to page limit.

Under our laboratory test conditions [41], it is found that the internal resistance is mainly affected by $T_{\text {in }}$ and it only increases slightly at a low SOC state. Therefore, we consider the internal resistance $R$ a function of the battery internal temperature $T_{\text {in }}$. The relationship for the internal resistance with different internal temperatures can be described as $R=R\left(T_{\text {in }}\right)$ and is shown in Table 2. Then, for different $T_{\text {in }}$ conditions, the corresponding $R$ will be calculated by the linear interpolation approach. 


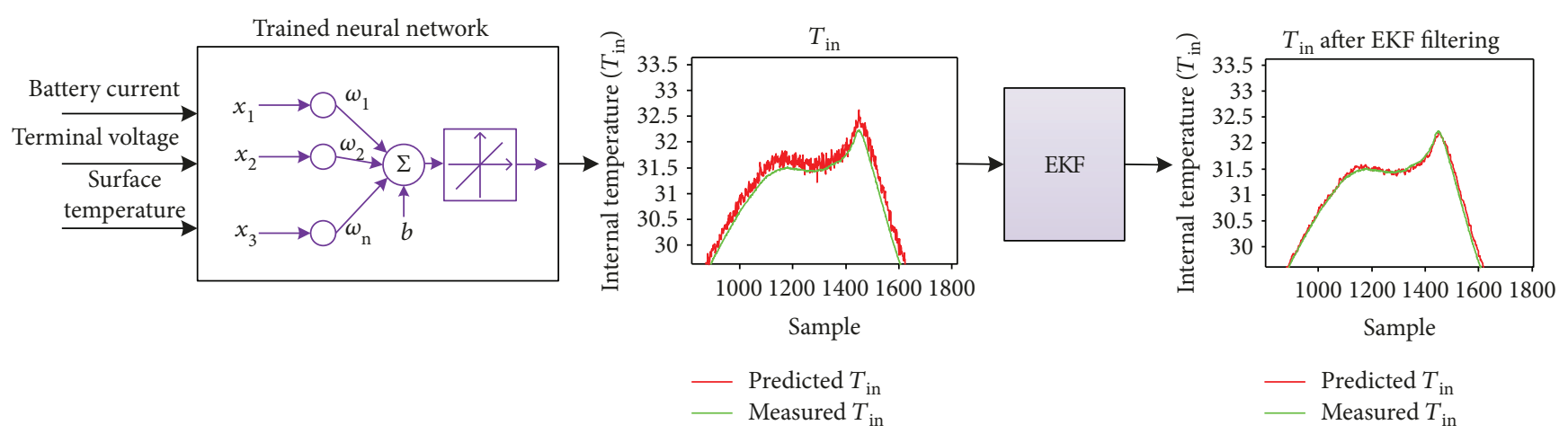

FIGURE 5: The developed approach combines the NN and EKF to estimate $T_{\text {in }}$.

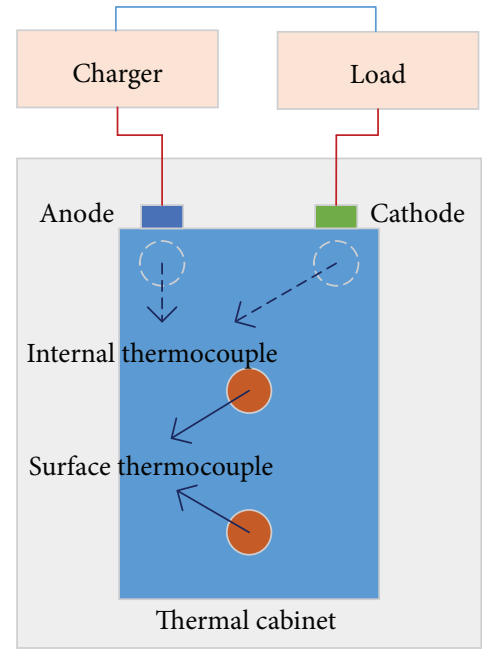

FIGURE 6: Schematic diagram of the battery operation platform (adapted from [41]).

TABLe 2: Battery $R$ under different $T_{\text {in }}$.

\begin{tabular}{cccccccc}
\hline$T_{\text {in }}\left({ }^{\circ} \mathrm{C}\right)$ & -10 & 0 & 10 & 23 & 32 & 39 & 52 \\
\hline$R(\mathrm{ohm})$ & 0.0261 & 0.0182 & 0.0165 & 0.0154 & 0.0124 & 0.0127 & 0.0118 \\
\hline
\end{tabular}

After $R$ is obtained, the constant parameters of the battery lumped thermal model will be identified by the least square method and are illustrated in Table 3. Then, the identified thermal model is determined as the state function with the EKF to filter outliers.

4.3. Determination of the Neural Network. The candidate input pool for the battery $\mathrm{NN}$ model is composed of $T_{\text {sh }}\left(k-k_{\mathrm{sh}}\right), V\left(k-k_{v}\right)$, and $I\left(k-k_{i}\right)$, where $T_{\mathrm{sh}}(k), V(k)$, and $I(k)$ stand for the surface temperature, terminal voltage, and current of the battery at a time instant $k$, respectively. $k_{\mathrm{sh}}$, $k_{v}$, and $k_{i}$ denote the delay factors of the corresponding elements, respectively. In the experiment, the delay factors are all fixed as 4 . Then, all varieties with delay up to 4 (the total number is 15) comprise the candidate pool. The elements in the candidate pool need to be evaluated by the TSIA approach.
TABLE 3: Constant thermal model parameters.

\begin{tabular}{lc}
\hline Constant model parameters & Value \\
\hline$C_{1}$ & 264.1 \\
$C_{2}$ & 30.8 \\
$k_{1}$ & 1.284 \\
$k_{2}$ & 0.301 \\
\hline
\end{tabular}

According to the TSIA approach, 7 terms from the candidate pool that make the most significant contributions to reduce the SSE are chosen as the input terms of the battery $\mathrm{NN}$ model, including $T_{\mathrm{sh}}(k), T_{\mathrm{sh}}(k-3), I(k)$, $I(k-3), V(k), V(k-1)$, and $V(k-3)$.

After selecting the satisfactory input terms, the number of the hidden neurons in the RBF NN model needs to be determined. The contributions of the hidden neurons are computed by the algorithm also named FRA in the forward selection stage of the TSIA. This process continues until the SSE criterion is achieved. In this experiment, the selected 7 inputs form a new term pool for determining the optimal number of neurons in the hidden layer. The initial number of the RBF neurons is preset as 25, and the stopped SSE percent value is set as $1 e^{-2}$ which means that the hidden neuron determination process is terminated on the condition that the total contribution of the selected hidden neurons is larger than the tolerance percent value $1-1 e^{-2}$. Then, the optimal number of neurons in the hidden layer of the RBF $\mathrm{NN}$ is finally determined as 12 , while the construction of the RBF NN model and the corresponding generalization capability are also determined and optimized. After that, the number of generations $G_{m}$ and the number of populations $N_{\mathrm{p}}$ for TLBO are set to 50 and 20 , respectively. The TLBO algorithm is then adopted to optimize the centres and widths in every neuron of basis function, while the linear output weights $W$ between the RBF hidden layer and output layer are calculated by the least square approach shown in (5).

4.4. Validation Results of the Hybrid Approach. In this study, four various testing data sets are conducted as the validation 


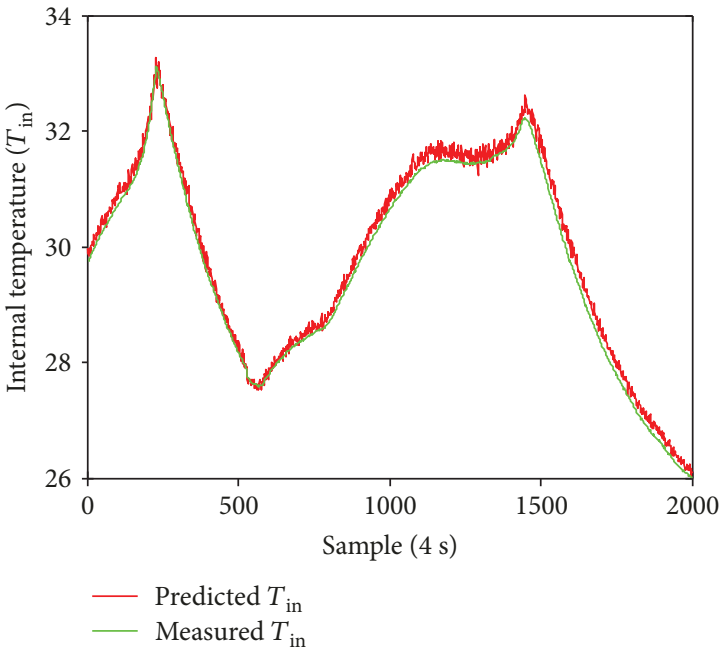

(a)

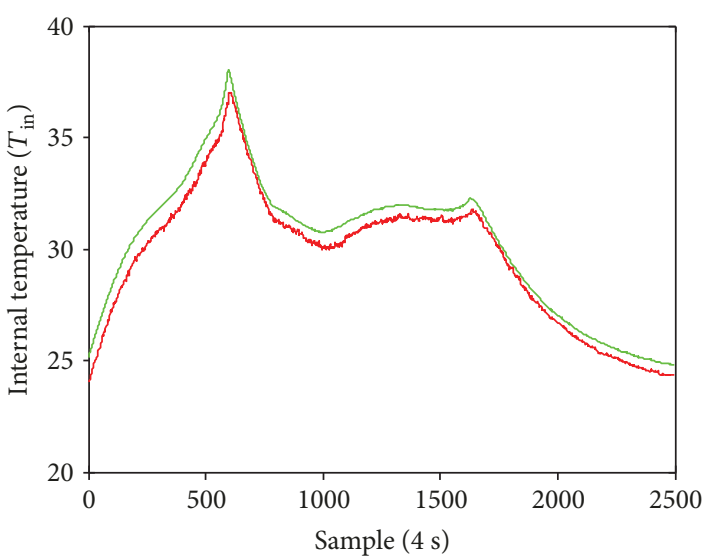

- Predicted $T_{\text {in }}$

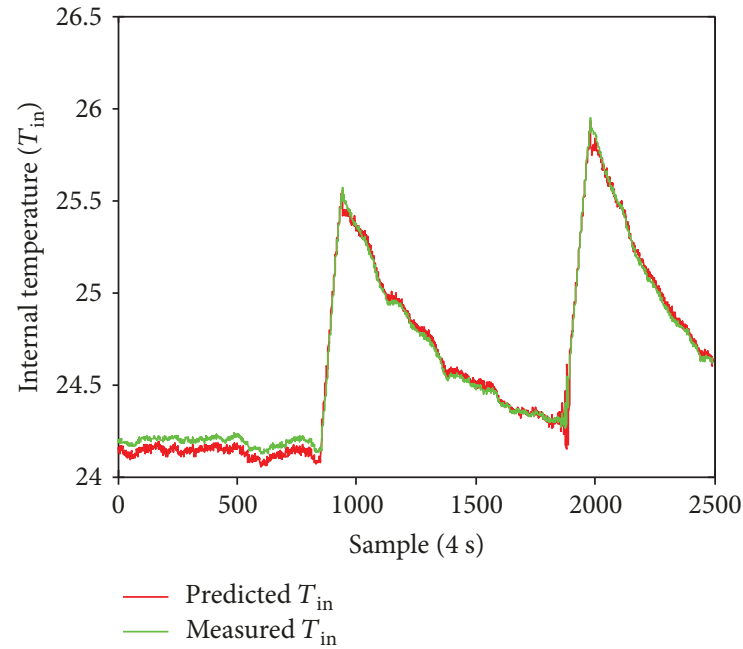

(b)

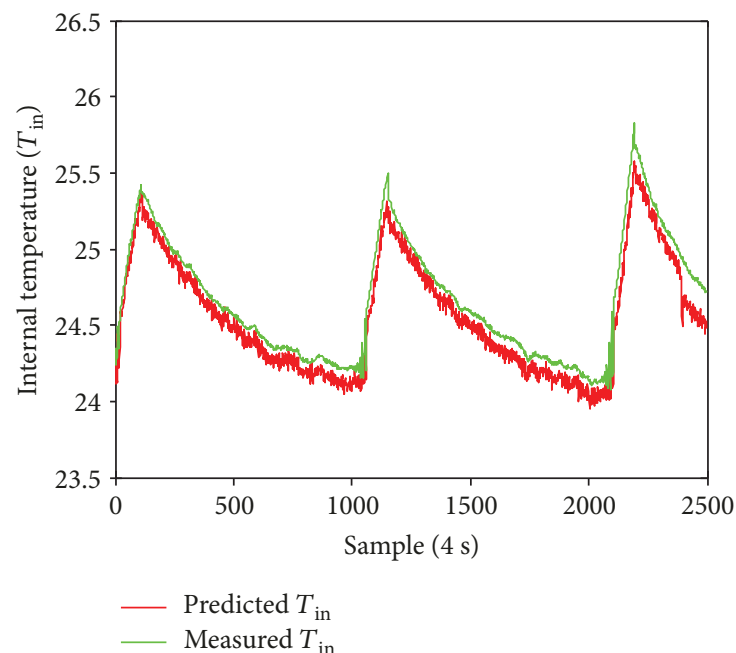

(d)

FIgURe 7: Validation results for $T_{\text {in }}$ estimation by using the solo linear NN model: (a) LC $-T_{\text {in }}$; (b) SC $-T_{\text {in }}$; (c) $L D-T_{\text {in }}$; (d) SD - $T_{\text {in }}$.

data, aimed at validating the robustness and the generalization performance of the well-trained NN model. All these testing data are obtained under $24^{\circ} \mathrm{C}$ constant ambient temperature. Then, the effectiveness of the RBF NN model is compared with that of the linear NN model.

Figure 7 illustrates the validation results of the battery $T_{\text {in }}$ estimation by the solo linear NN model under four different test cases. Figure 7 (a) shows the test data with $T_{\text {in }}$ that varied in a relatively large range under the charging condition denoted as LC $-T_{\text {in }}$, and Figure 7(b) illustrates another test data with $T_{\text {in }}$ that varied in a small range under the charging condition denoted as $\mathrm{SC}-T_{\text {in }}$; Figure 7 (c) shows the discharging test data with $T_{\text {in }}$ that varied in a relatively large range denoted as $\mathrm{LD}-T_{\mathrm{in}}$, while Figure $7(\mathrm{~d})$ illustrates another discharging test data with $T_{\text {in }}$ that varied in a small range denoted as $\mathrm{SD}-T_{\mathrm{in}}$. Because the temperature variation in the battery is a relatively slow process in comparison with terminal voltage and battery current, which means that battery temperature cannot change dramatically within a
TABLE 4: Estimation errors for four validation data by using the solo linear NN model.

\begin{tabular}{lcc}
\hline Case & RMSE & Max error $\left({ }^{\circ} \mathrm{C}\right)$ \\
\hline $\mathrm{LC}-T_{\text {in }}(\mathrm{a})$ & 0.1801 & 0.458 \\
$\mathrm{SC}-T_{\text {in }}(\mathrm{b})$ & 0.1262 & 0.221 \\
$\mathrm{LD}-T_{\text {in }}(\mathrm{c})$ & 0.5645 & 1.302 \\
$\mathrm{SD}-T_{\text {in }}(\mathrm{d})$ & 0.1995 & 0.325 \\
\hline
\end{tabular}

very short sample period (e.g., $1 \mathrm{~s}$ ), therefore, the sampling time $T_{\mathrm{s}}$ in all these data are set as $4 \mathrm{~s}$. The red line stands for the estimated battery $T_{\text {in }}$ by the solo linear NN model, while the green line denotes the measured battery $T_{\text {in }}$ by the thermocouple in the experiment. The estimation errors for these four validated data by using the solo linear NN model are shown in Table 4. It can be seen clearly that for charging conditions, the RMSE is within 0.2 and the maximum estimated errors are within $0.5^{\circ} \mathrm{C}$ for both $\mathrm{LC}-T_{\text {in }}$ 


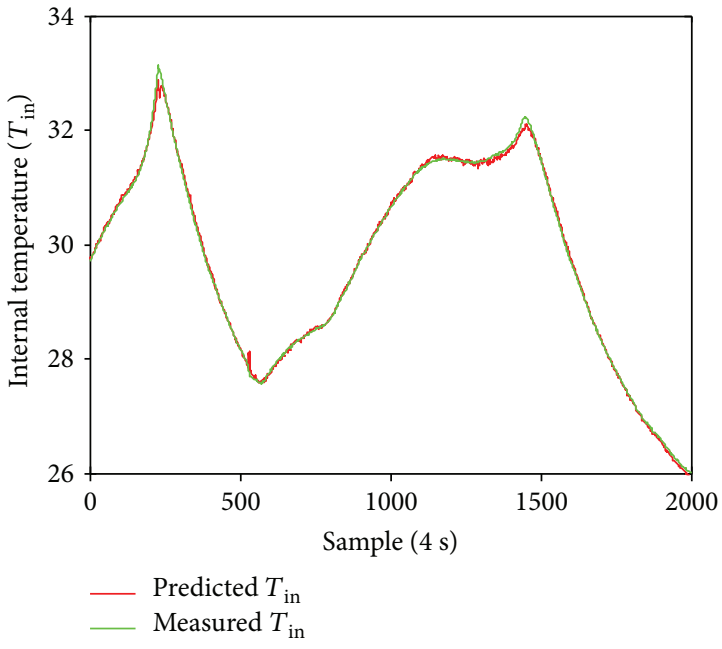

(a)

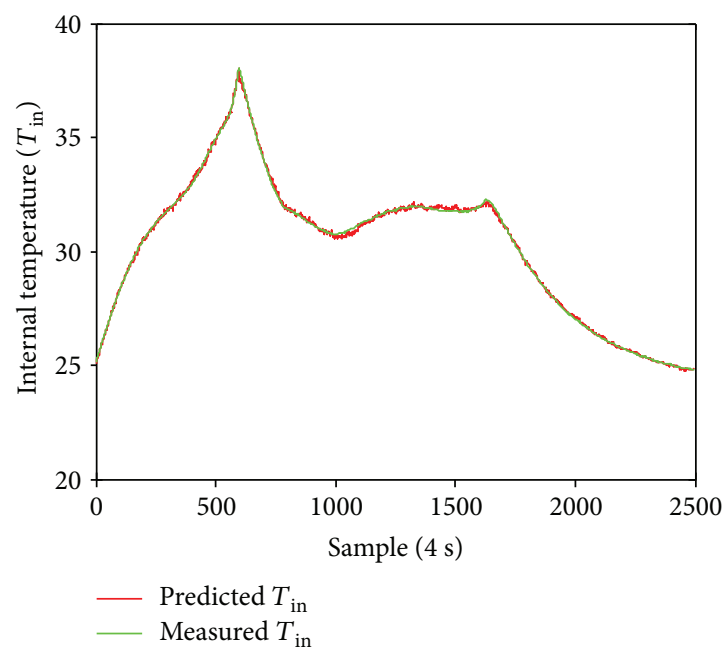

(c)

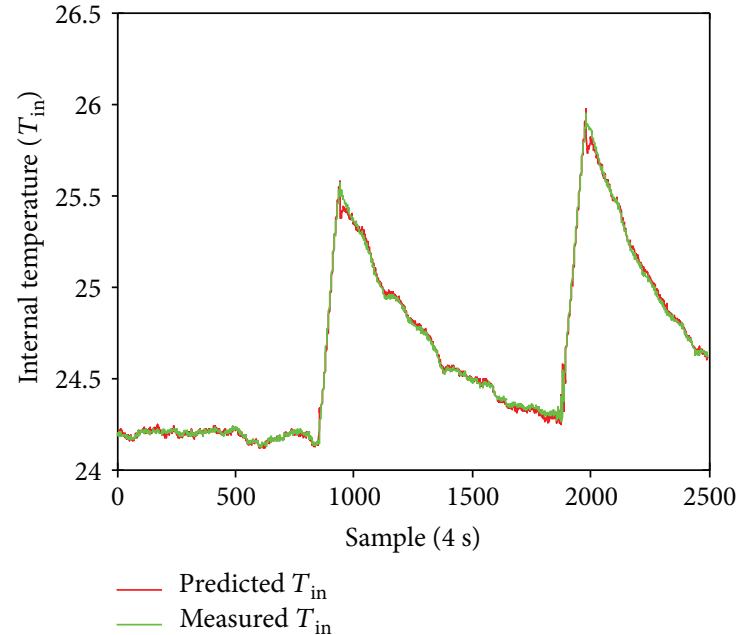

(b)

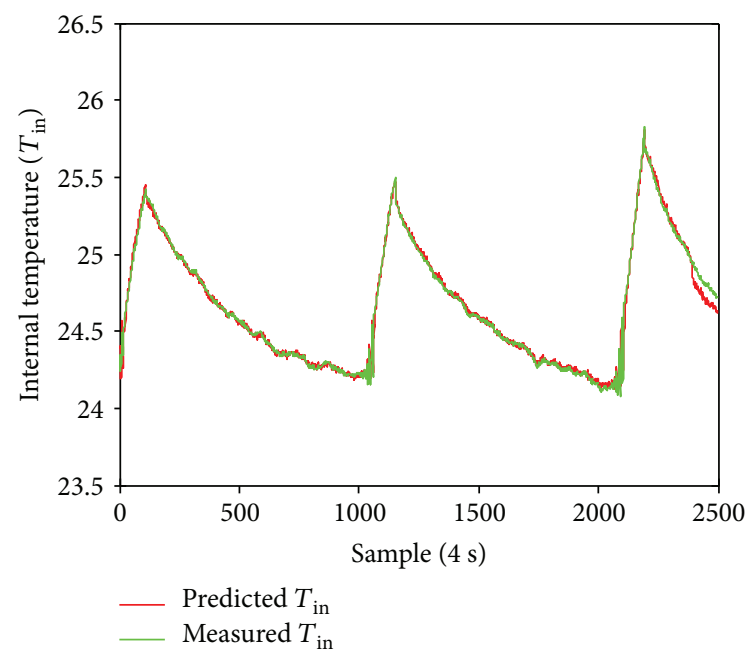

(d)

FIgURE 8: Validation results for $T_{\text {in }}$ estimation by using the solo RBF NN model: (a) LC $-T_{\text {in }}$; (b) SC $-T_{\text {in }}$; (c) $L D-T_{\text {in }}$; (d) SD - $T_{\text {in }}$.

and SC $-T_{\text {in }}$. These errors primarily appear with the form of fluctuant noises especially in some inflection points. In discharging conditions, the RMSE is 0.5645 while the maximum error is over $1.3^{\circ} \mathrm{C}$ for the $\mathrm{LD}-T_{\text {in }}$, which is not accurate enough for estimating the battery $T_{\mathrm{in}}$. Besides, more fluctuant noises appear for the $\mathrm{SD}-T_{\text {in }}$; this result illustrates that estimating the battery $T_{\text {in }}$ using only the solo linear NN model may lose its generalization ability under different conditions.

As discussed in the previous section, the trained RBF NN model is also applied for battery internal temperature estimation. Figure 8 illustrates the validation results by using the solo RBF NN model to estimate the battery $T_{\text {in }}$ under four test cases. It can be seen from the figures that the trained RBF NN model captures the evolution of most $T_{\text {in }}$ for all conditions. Table 5 illustrates the estimation errors by using the solo trained RBF NN model. In charging conditions, the RMSE reduces to 0.0475 and 0.0193 for the $L C-T_{\text {in }}$ and $\mathrm{SC}-T_{\text {in }}$, respectively, which is more accurate than the results by using the solo linear NN model. The errors are less
TABLE 5: Estimation errors for four validation data by using the solo RBF NN model.

\begin{tabular}{lcc}
\hline Case & RMSE & Max error $\left({ }^{\circ} \mathrm{C}\right)$ \\
\hline $\mathrm{LC}-T_{\text {in }}(\mathrm{a})$ & 0.0475 & 0.431 \\
$\mathrm{SC}-T_{\text {in }}(\mathrm{b})$ & 0.0193 & 0.233 \\
$\mathrm{LD}-T_{\text {in }}(\mathrm{c})$ & 0.0939 & 0.366 \\
$\mathrm{SD}-T_{\text {in }}(\mathrm{d})$ & 0.0367 & 0.192 \\
\hline
\end{tabular}

than $0.2^{\circ} \mathrm{C}$ for most temperature points except a few inflection points with maximum errors up to $0.4^{\circ} \mathrm{C}$. In discharging conditions, the RMSE for the cases of LC $-T_{\text {in }}$ and SC $-T_{\text {in }}$ are only 0.0939 and 0.0367 , while the maximum errors reduce to $0.366^{\circ} \mathrm{C}$ and $0.192^{\circ} \mathrm{C}$, respectively. Validation results demonstrate that the established RBF NN model captures the battery $T_{\text {in }}$ behaviours with great accuracy and robustness except a few fluctuant noises happening in inflection points. 


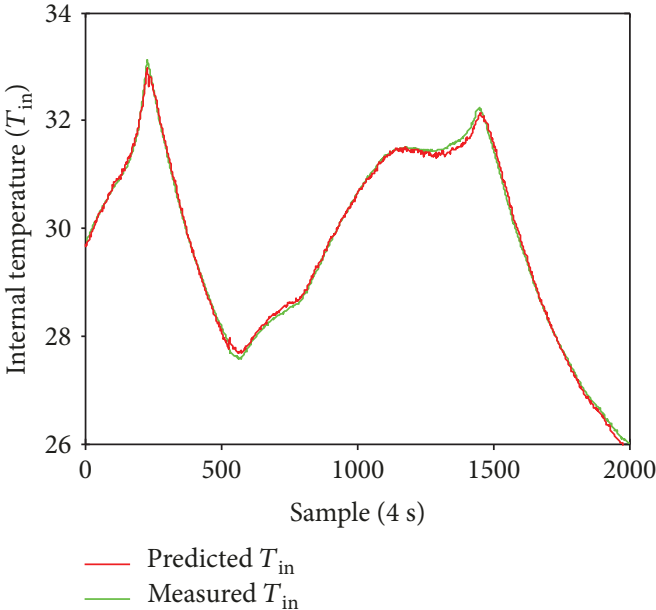

(a)

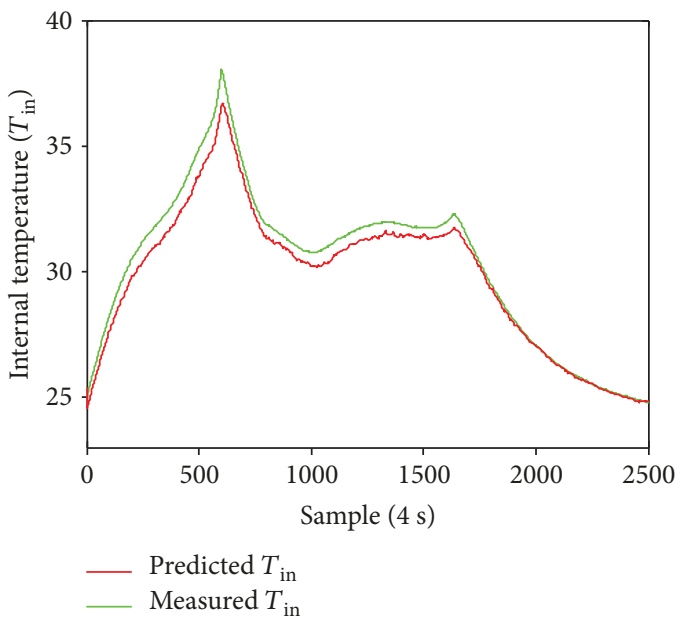

(c)

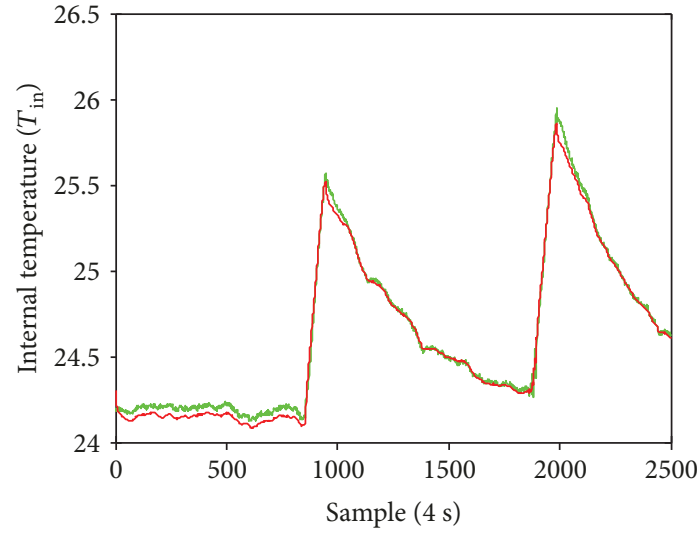

— Predicted $T_{\mathrm{in}}$ Measured $T_{\text {in }}$

(b)

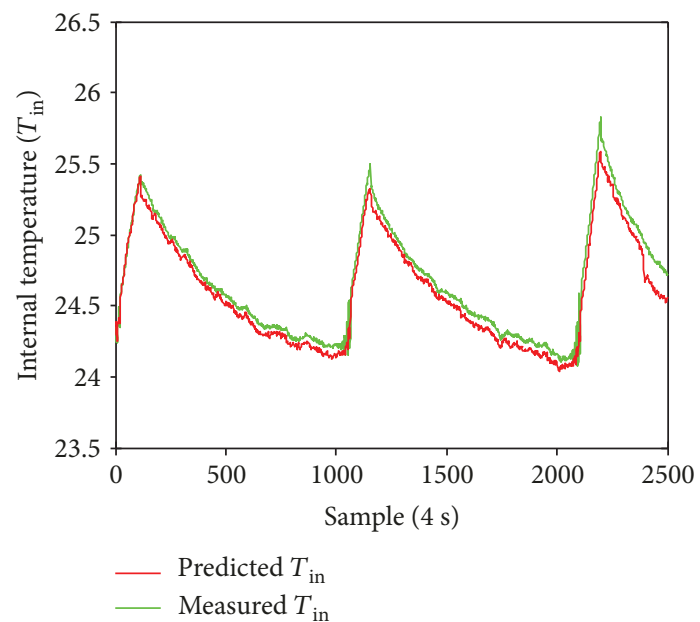

(d)

FIGURE 9: Validation results for $T_{\text {in }}$ estimation by combining the linear NN and EKF: (a) $L C-T_{\text {in }}$; (b) $S C-T_{\text {in }}$; (c) $L D-T_{\text {in }}$; (d) SD $-T_{\text {in }}$.

TABLE 6: Estimation errors for four validation data by combining the linear NN and EKF.

\begin{tabular}{lcc}
\hline Temperature range & RMSE & Max error $\left({ }^{\circ} \mathrm{C}\right)$ \\
\hline $\mathrm{LC}-T_{\text {in }}(\mathrm{a})$ & 0.0809 & 0.267 \\
$\mathrm{SC}-T_{\text {in }}(\mathrm{b})$ & 0.0746 & 0.202 \\
$\mathrm{LD}-T_{\text {in }}(\mathrm{c})$ & 0.5251 & 1.242 \\
$\mathrm{SD}-T_{\text {in }}(\mathrm{d})$ & 0.1246 & 0.297 \\
\hline
\end{tabular}

As discussed in Section 3.3, the EKF is adopted to filter the outliers in the battery NN models. Figure 9 illustrates the filtering results by combining the linear NN model and EKF under four different conditions. It is clear that the fluctuant outliers can be filtered out by using the EKF. Table 6 illustrates the estimation errors for four validation data by combining the linear NN model and the EKF algorithm. Both the RMSE and maximum errors are reduced by using the combined method compared with the solo linear
$\mathrm{NN}$ model estimation. For the result of $\mathrm{LC}-T_{\text {in }}$ validation, the RMSE is 0.1801 while the maximum error reaches $0.458^{\circ} \mathrm{C}$ by the solo linear battery NN model. After using the EKF to filter out the outliers, the RMSE decreases to 0.0809 that is nearly half of the former, while the maximum error reduces to $0.267^{\circ} \mathrm{C}$. For the result of $\mathrm{SC}-T_{\text {in }}$ validation, the RMSE is 0.1262 and the maximum error is $0.221^{\circ} \mathrm{C}$ by using the solo linear NN model. By combining the linear NN model and the EKF algorithm, the RMSE and the maximum error both decrease to 0.0746 and $0.202^{\circ} \mathrm{C}$, respectively. In discharging conditions, the RMSE and the maximum error are 0.5251 and $1.242^{\circ} \mathrm{C}$, respectively, for the $\mathrm{LD}-T_{\text {in }}$ after using the EKF, while the RMSE and the maximum error are 0.1246 and $0.297^{\circ} \mathrm{C}$, respectively, for the SD $-T_{\text {in }}$. Therefore, the approach combining the linear $\mathrm{NN}$ model and the EKF algorithm is effective in filtering out the outliers and in reducing the $T_{\text {in }}$ estimation error in the linear NN model. It should be known that both the RMSE and maximum error of the validation results will decrease by using the EKF, but the $T_{\text {in }}$ estimated results also seem 


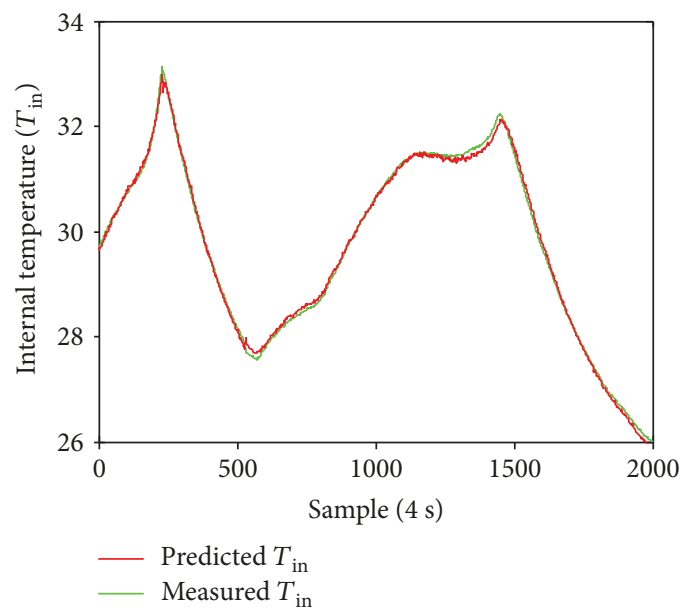

(a)

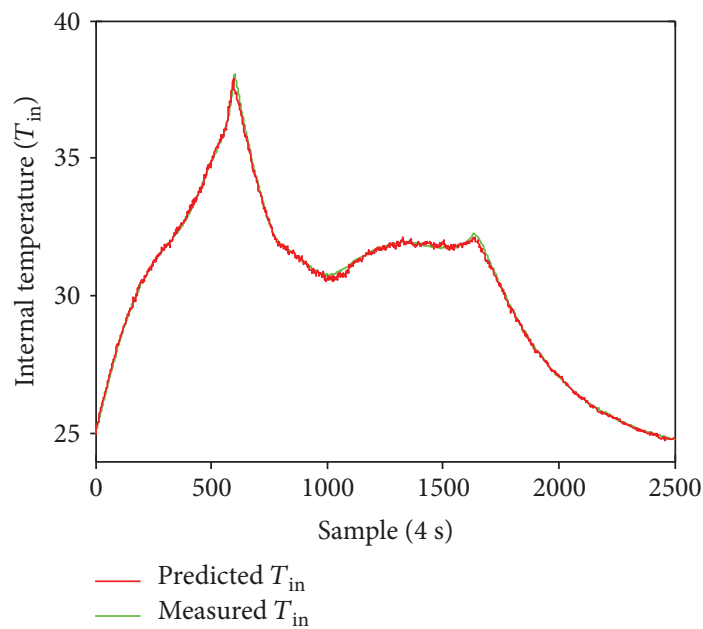

(c)

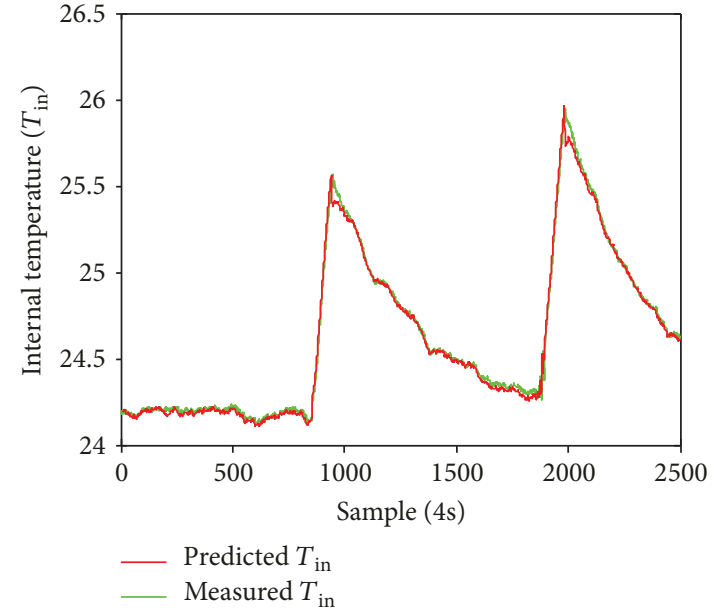

(b)

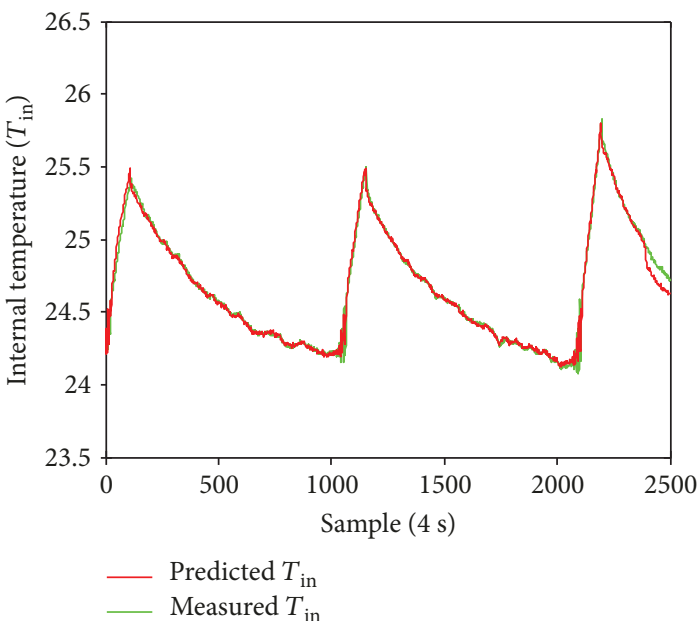

(d)

FIgURE 10: Validation results for $T_{\text {in }}$ estimation by combining the RBF NN and EKF: (a) LC $-T_{\text {in }}$; (b) $S C-T_{\text {in }}$; (c) $L D-T_{\text {in }}$; (d) SD - $T_{\text {in }}$.

to be not accurate enough due to the bad robustness and generalization ability of the linear NN model.

The EKF is also applied to verify the $T_{\text {in }}$ estimation performance of the RBF NN model. The battery $T_{\text {in }}$ estimation results for four different cases by using the approach combining the RBF NN model and the EKF are shown in Figure 10. The corresponding estimation errors are also illustrated in Table 7. It can be seen from Figure 10 that the EKF captures the evolution process of battery $T_{\text {in }}$ for all cases, and most fluctuant noises are successfully filtered out by the EKF algorithm. In charging conditions, the results show that the RMSE between actual $T_{\text {in }}$ and estimated $T_{\text {in }}$ for $L C-T_{\text {in }}$ is 0.0407 - an improvement by $14 \%$, and that for $\mathrm{SC}-T_{\text {in }}$ is 0.0154 - an improvement by $20 \%$, relative to the solo RBF $\mathrm{NN}$ model. The maximum error for the EKF could be within $0.25^{\circ} \mathrm{C}$. In discharging conditions, the RMSE for $\mathrm{LD}-T_{\text {in }}$ after the EKF is 0.0647 -an improvement by $31 \%$, and that for $\mathrm{SD}-T_{\text {in }}$ after the EKF is 0.0294-an improvement by $20 \%$. The maximum errors could be within $0.25^{\circ} \mathrm{C}$, reflecting that the hybrid approach combining the RBF NN model and EKF has higher $T_{\text {in }}$ estimation accuracy compared with the
TABLE 7: Estimation errors for four validation data by combining the RBF NN and EKF.

\begin{tabular}{lcc}
\hline Case & RMSE & Max error $\left({ }^{\circ} \mathrm{C}\right)$ \\
\hline $\mathrm{LC}-T_{\text {in }}(\mathrm{a})$ & 0.0407 & 0.221 \\
$\mathrm{SC}-T_{\text {in }}(\mathrm{b})$ & 0.0154 & 0.194 \\
$\mathrm{LD}-T_{\text {in }}(\mathrm{c})$ & 0.0647 & 0.243 \\
$\mathrm{SD}-T_{\text {in }}(\mathrm{d})$ & 0.0294 & 0.168 \\
\hline
\end{tabular}

solo RBF NN model. Therefore, combining the well-trained RBF NN model and the EKF should be an effective method to filter out the fluctuant noises and reduce the errors for battery $T_{\text {in }}$ estimation. However, a more accurate $T_{\text {in }}$ estimation using the EKF requires accurate parameters for a battery state model, which limits its wide application. So to select the hybrid approach combining the trained RBF NN model and EKF or just the solo trained RBF NN model for battery $T_{\text {in }}$ estimation should be highly dependent on the required accuracy for battery application. 


\section{Conclusion}

In this paper, a data-driven approach is developed in the application of estimating the internal temperature for Li-ion battery thermal management. This novel approach combines a well-trained RBF NN model and the EKF algorithm, which is distinctively different from existing methods. In order to capture the dynamics of the battery, multiple measurements including battery current, terminal voltage, and surface temperature are chosen as the inputs of the RBF NN, while battery internal temperature is chosen as the output. In order to determine the suitable inputs and remove the superfluous terms to prevent overfitting and decrease the cost of computation, a TSIA is first adopted to select the most significant inputs. And the number of RBF hidden neurons is also determined by the FRA in the forward selection stage of the TSIA, where the structure of the RBF $\mathrm{NN}$ is optimized to achieve satisfied generalization capability. To reduce the estimation errors and further increase the accuracy of battery $T_{\text {in }}$ estimation, a lumped thermal model is applied as the state function with the EKF to filter out the outliers in the NN model. The experiments demonstrate that the proposed RBF NN-based hybrid $T_{\text {in }}$ approach achieves the reliable and accurate estimation of the battery internal temperature under different conditions. By using the EKF, the RMSE of estimation is within 0.07 , while the maximum error is less than $0.25^{\circ} \mathrm{C}$ for various testing conditions.

The contributions of this work are mainly threefold: (1) a two-stage stepwise identification algorithm is adopted to select the proper inputs and determine the optimal number of the hidden neurons of the RBF NN, where the structure of the RBF NN model is optimized to achieve satisfactory performance. (2) A novel state-space model combining the lumped thermal model and the well-trained battery $\mathrm{NN}$ model is proposed. Moreover, the EKF algorithm is applied to filter out the outliers of the solo NN model and decrease the estimation errors, and the accuracy for battery internal temperature estimation under different conditions is improved. (3) The NN is a data-driven approach without the requirement of any battery background knowledge, so the developed hybrid approach is capable of estimating other types of batteries conveniently to achieve reasonable thermal management.

\section{Data Availability}

The data used to support the findings of this study are available from the corresponding author upon request.

\section{Conflicts of Interest}

The authors declare that they have no conflicts of interest.

\section{Acknowledgments}

This work was partially funded by the UK EPSRC under Grant "intelligent grid interfaced vehicle eco-charging (iGIVE)" EP/L001063/1 and the NSFC under Grants
51607177, 61533010, 61673256, and U1435215. Kailong Liu would like to thank the EPSRC for sponsoring his research.

\section{References}

[1] A. B. Gallo, J. R. Simões-Moreira, H. K. M. Costa, M. M. Santos, and E. Moutinho dos Santos, "Energy storage in the energy transition context: a technology review," Renewable and Sustainable Energy Reviews, vol. 65, pp. 800-822, 2016.

[2] K. Liu, K. Li, Q. Peng, and C. Zhang, "A brief review on key technologies in the battery management system of electric vehicles," Frontiers of Mechanical Engineering, pp. 1-18, 2018.

[3] M. A. Hannan, M. M. Hoque, A. Mohamed, and A. Ayob, "Review of energy storage systems for electric vehicle applications: issues and challenges," Renewable and Sustainable Energy Reviews, vol. 69, pp. 771-789, 2017.

[4] Z. Yang, K. Li, Q. Niu, and Y. Xue, "A comprehensive study of economic unit commitment of power systems integrating various renewable generations and plug-in electric vehicles," Energy Conversion and Management, vol. 132, pp. 460481, 2017.

[5] G. Xia, L. Cao, and G. Bi, "A review on battery thermal management in electric vehicle application," Journal of Power Sources, vol. 367, pp. 90-105, 2017.

[6] M. Berecibar, I. Gandiaga, I. Villarreal, N. Omar, J. van Mierlo, and P. van den Bossche, "Critical review of state of health estimation methods of Li-ion batteries for real applications," Renewable and Sustainable Energy Reviews, vol. 56, pp. 572587, 2016.

[7] C. Zou, C. Manzie, D. Nešić, and A. G. Kallapur, "Multi-timescale observer design for state-of-charge and state-of-health of a lithium-ion battery," Journal of Power Sources, vol. 335, pp. 121-130, 2016.

[8] Y. Tian, D. Li, J. Tian, and B. Xia, "State of charge estimation of lithium-ion batteries using an optimal adaptive gain nonlinear observer," Electrochimica Acta, vol. 225, pp. 225-234, 2017.

[9] M. A. Hannan, M. S. H. Lipu, A. Hussain, and A. Mohamed, "A review of lithium-ion battery state of charge estimation and management system in electric vehicle applications: challenges and recommendations," Renewable and Sustainable Energy Reviews, vol. 78, pp. 834-854, 2017.

[10] K. Liu, K. Li, Z. Yang, C. Zhang, and J. Deng, “An advanced lithium-ion battery optimal charging strategy based on a coupled thermoelectric model," Electrochimica Acta, vol. 225, pp. 330-344, 2017.

[11] M. Wang and H. X. Li, "Real-time estimation of temperature distribution for cylindrical lithium-ion batteries under boundary cooling," IEEE Transactions on Industrial Electronics, vol. 64, no. 3, pp. 2316-2324, 2017.

[12] G. Zhang, S. Ge, T. Xu, X. G. Yang, H. Tian, and C. Y. Wang, "Rapid self-heating and internal temperature sensing of lithium-ion batteries at low temperatures," Electrochimica Acta, vol. 218, pp. 149-155, 2016.

[13] C. Martinez-Cisneros, C. Antonelli, B. Levenfeld, A. Varez, and J. Y. Sanchez, "Evaluation of polyolefin-based macroporous separators for high temperature Li-ion batteries," Electrochimica Acta, vol. 216, pp. 68-78, 2016.

[14] K. Liu, K. Li, H. Ma, J. Zhang, and Q. Peng, "Multi-objective optimization of charging patterns for lithium-ion battery management," Energy Conversion and Management, vol. 159, pp. 151-162, 2018. 
[15] Q. Sun, Q. Wang, X. Zhao, J. Sun, and Z. Lin, "Numerical study on lithium titanate battery thermal response under adiabatic condition," Energy Conversion and Management, vol. 92, pp. 184-193, 2015.

[16] C. Y. Lee, S. J. Lee, Y. M. Hung et al., "Integrated microsensor for real-time microscopic monitoring of local temperature, voltage and current inside lithium ion battery," Sensors and Actuators A: Physical, vol. 253, pp. 59-68, 2017.

[17] Y. Kim, S. Mohan, J. B. Siegel, A. G. Stefanopoulou, and Y. Ding, "The estimation of temperature distribution in cylindrical battery cells under unknown cooling conditions," IEEE Transactions on Control Systems Technology, vol. 22, no. 6, pp. 2277-2286, 2014.

[18] X. Lin, H. E. Perez, S. Mohan et al., "A lumped-parameter electro-thermal model for cylindrical batteries," Journal of Power Sources, vol. 257, pp. 1-11, 2014.

[19] K. Liu, K. Li, and C. Zhang, "Constrained generalized predictive control of battery charging process based on a coupled thermoelectric model," Journal of Power Sources, vol. 347, pp. 145-158, 2017.

[20] E. Samadani, S. Farhad, W. Scott et al., "Empirical modeling of lithium-ion batteries based on electrochemical impedance spectroscopy tests," Electrochimica Acta, vol. 160, pp. 169177, 2015.

[21] R. R. Richardson, P. T. Ireland, and D. A. Howey, "Battery internal temperature estimation by combined impedance and surface temperature measurement," Journal of Power Sources, vol. 265, pp. 254-261, 2014.

[22] R. R. Richardson and D. A. Howey, "Sensorless battery internal temperature estimation using a Kalman filter with impedance measurement," IEEE Transactions on Sustainable Energy, vol. 6, no. 4, pp. 1190-1199, 2015.

[23] C. Sbarufatti, M. Corbetta, M. Giglio, and F. Cadini, "Adaptive prognosis of lithium-ion batteries based on the combination of particle filters and radial basis function neural networks," Journal of Power Sources, vol. 344, pp. 128-140, 2017.

[24] J. Chen, Q. Ouyang, C. Xu, and H. Su, "Neural network-based state of charge observer design for lithium-ion batteries," IEEE Transactions on Control Systems Technology, vol. 26, no. 1, pp. 313-320, 2018.

[25] L. Zhang, K. Li, Z. Yang et al., "Compact neural modelling of single flow zinc-nickel batteries based on Jaya optimization," in 2018 IEEE Congress on Evolutionary Computation (CEC), pp. 1-6, Brazil, July 2018.

[26] Z. Deng, L. Yang, Y. Cai, and H. Deng, "Maximum available capacity and energy estimation based on support vector machine regression for lithium-ion battery," Energy Procedia, vol. 107, pp. 68-75, 2017.

[27] R. Khelif, B. Chebel-Morello, S. Malinowski, E. Laajili, F. Fnaiech, and N. Zerhouni, "Direct remaining useful life estimation based on support vector regression," IEEE Transactions on Industrial Electronics, vol. 64, no. 3, pp. 2276-2285, 2017.

[28] K. Liu, K. Li, and J. Deng, "A novel hybrid data-driven method for Li-ion battery internal temperature estimation," in 2016 UKACC 11th International Conference on Control (CONTROL), pp. 1-6, Belfast, UK, 2016, IEEE.

[29] K. Li, J. X. Peng, and E. W. Bai, "A two-stage algorithm for identification of nonlinear dynamic systems," Automatica, vol. 42, no. 7, pp. 1189-1197, 2006.
[30] K. Li, J.-X. Peng, and G. W. Irwin, “A fast nonlinear model identification method," IEEE Transactions on Automatic Control, vol. 50, no. 8, pp. 1211-1216, 2005.

[31] R. Rao, "Review of applications of TLBO algorithm and a tutorial for beginners to solve the unconstrained and constrained optimization problems," Decision Science Letters, vol. 5, no. 1, pp. 1-30, 2016.

[32] Y. Guo, K. Li, Z. Yang, J. Deng, and D. M. Laverty, “A novel radial basis function neural network principal component analysis scheme for PMU-based wide-area power system monitoring," Electric Power Systems Research, vol. 127, pp. 197205, 2015.

[33] Q. Zhang and F. Sepulveda, "RBFNN-based modelling and analysis for the signal reconstruction of peripheral nerve tissue," in Proceedings of the 8th ACM International Conference on Bioinformatics, Computational Biology, and Health Informatics - $A C M-B C B$ '17, pp. 474-479, Boston, MA, USA, 2017, ACM.

[34] X. Hong, R. J. Mitchell, S. Chen, C. J. Harris, K. Li, and G. W. Irwin, "Model selection approaches for non-linear system identification: a review," International Journal of Systems Science, vol. 39, no. 10, pp. 925-946, 2008.

[35] Z. Yang, K. Li, Q. Niu, Y. Xue, and A. Foley, “A self-learning TLBO based dynamic economic/environmental dispatch considering multiple plug-in electric vehicle loads," Journal of Modern Power Systems and Clean Energy, vol. 2, no. 4, pp. 298-307, 2014.

[36] R. V. Rao, V. J. Savsani, and D. P. Vakharia, "Teaching-learning-based optimization: a novel method for constrained mechanical design optimization problems," Computer-Aided Design, vol. 43, no. 3, pp. 303-315, 2011.

[37] H. Ma, P. You, K. Liu, Z. Yang, and M. Fei, “Optimal battery charging strategy based on complex system optimization," in Advanced Computational Methods in Energy, Power, Electric Vehicles, and Their Integration, pp. 371-378, Springer, Singapore, 2017.

[38] K. Liu, K. Li, Z. Yang, C. Zhang, and J. Deng, "Battery optimal charging strategy based on a coupled thermoelectric model," in 2016 IEEE Congress on Evolutionary Computation (CEC), pp. 5084-5091, Vancouver, BC, Canada, 2016, IEEE.

[39] Q. Wang, B. Jiang, B. Li, and Y. Yan, “A critical review of thermal management models and solutions of lithium-ion batteries for the development of pure electric vehicles," Renewable and Sustainable Energy Reviews, vol. 64, pp. 106-128, 2016.

[40] Y. Zhou, Q. Zhang, H. Wang, P. Zhou, and T. Chai, "EKFbased enhanced performance controller design for nonlinear stochastic systems," IEEE Transactions on Automatic Control, vol. 63, no. 4, pp. 1155-1162, 2018.

[41] C. Zhang, K. Li, and J. Deng, "Real-time estimation of battery internal temperature based on a simplified thermoelectric model," Journal of Power Sources, vol. 302, pp. 146-154, 2016. 


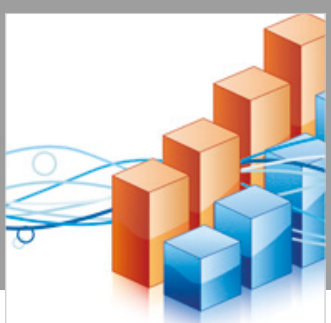

Advances in

Operations Research

\section{-n-m}
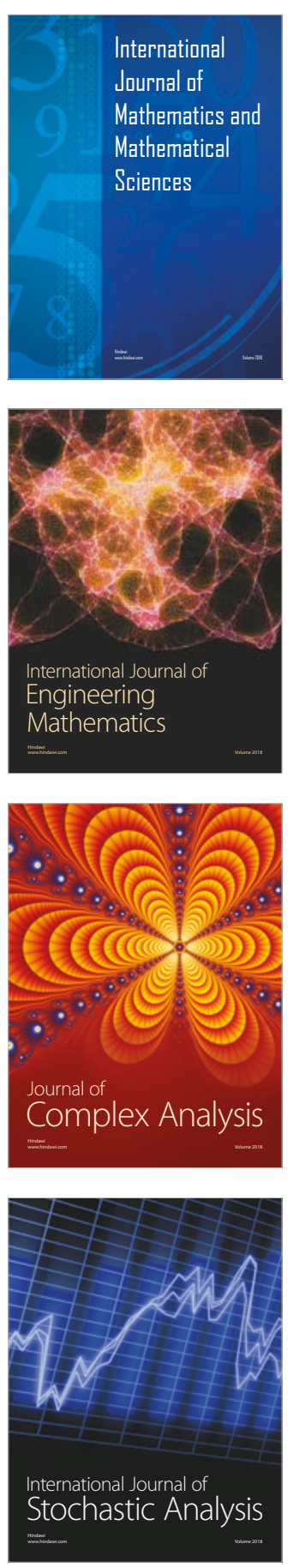
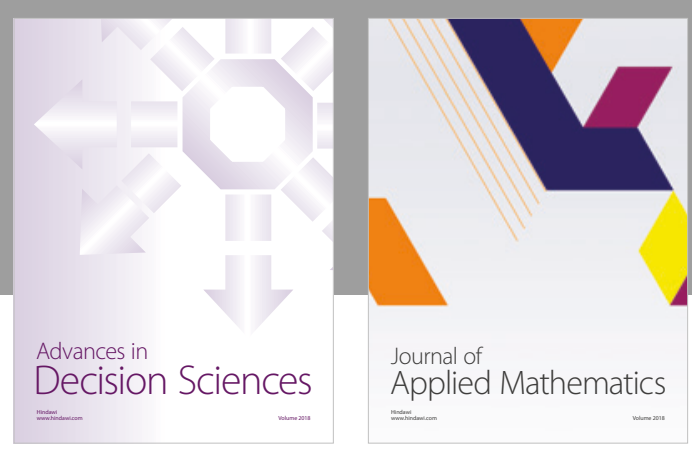

Journal of

Applied Mathematics
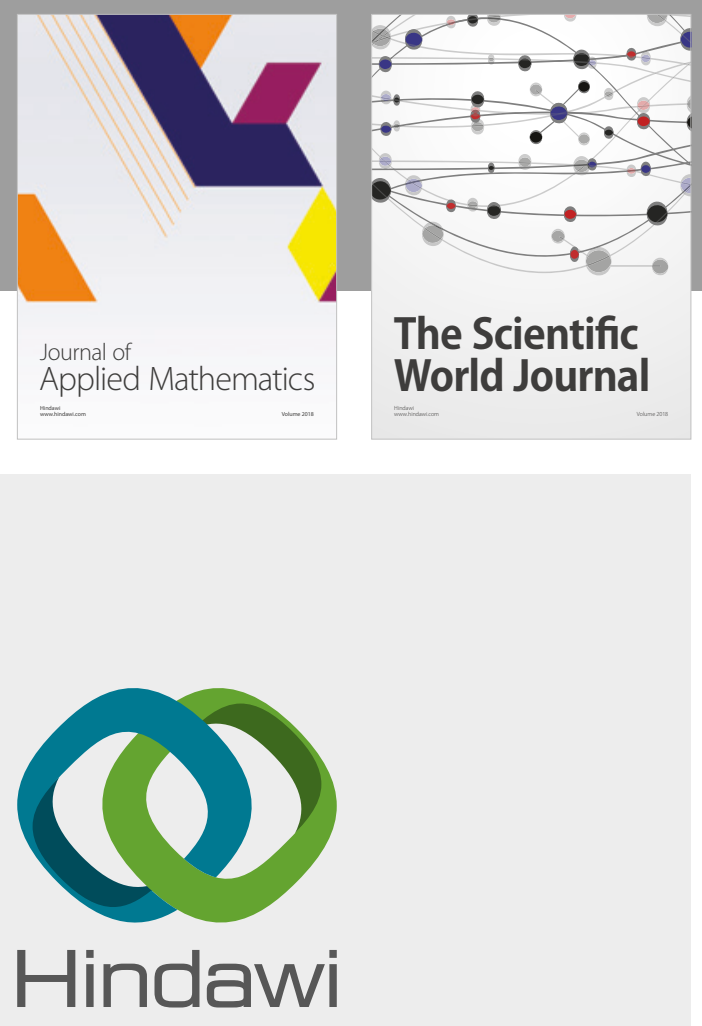

Submit your manuscripts at

www.hindawi.com

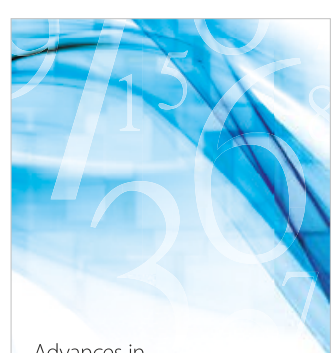

Advances in
Numerical Analysis
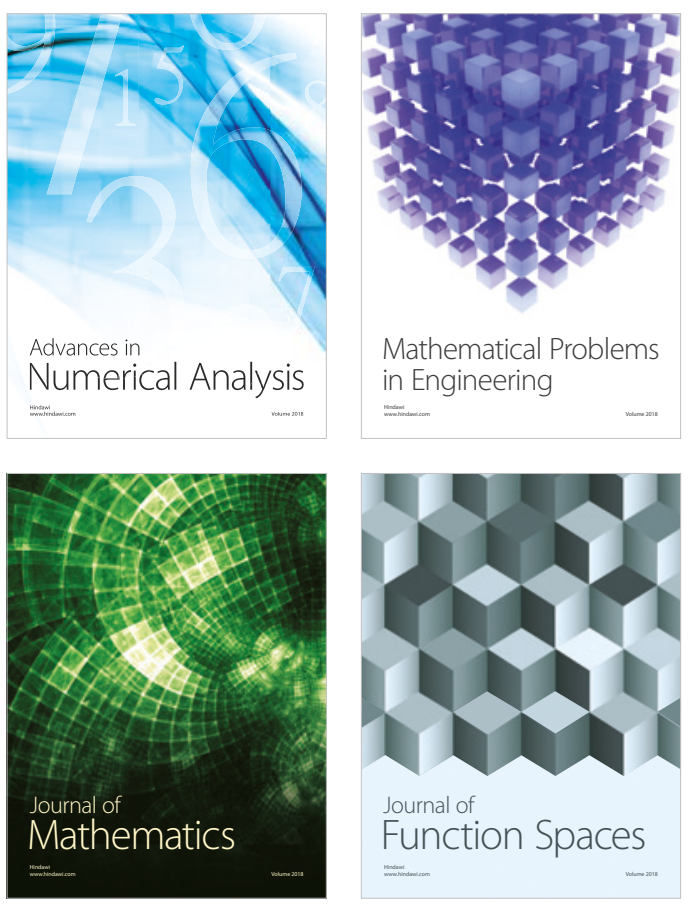

Mathematical Problems in Engineering

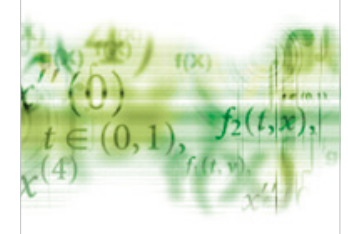

International Journal of

Differential Equations

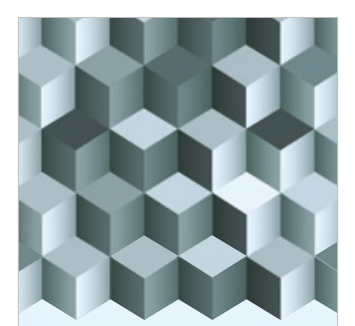

Journal of

Function Spaces
The Scientific

World Journal

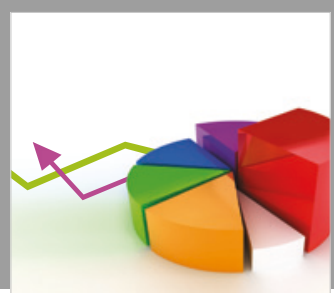

Journal of

Probability and Statistics
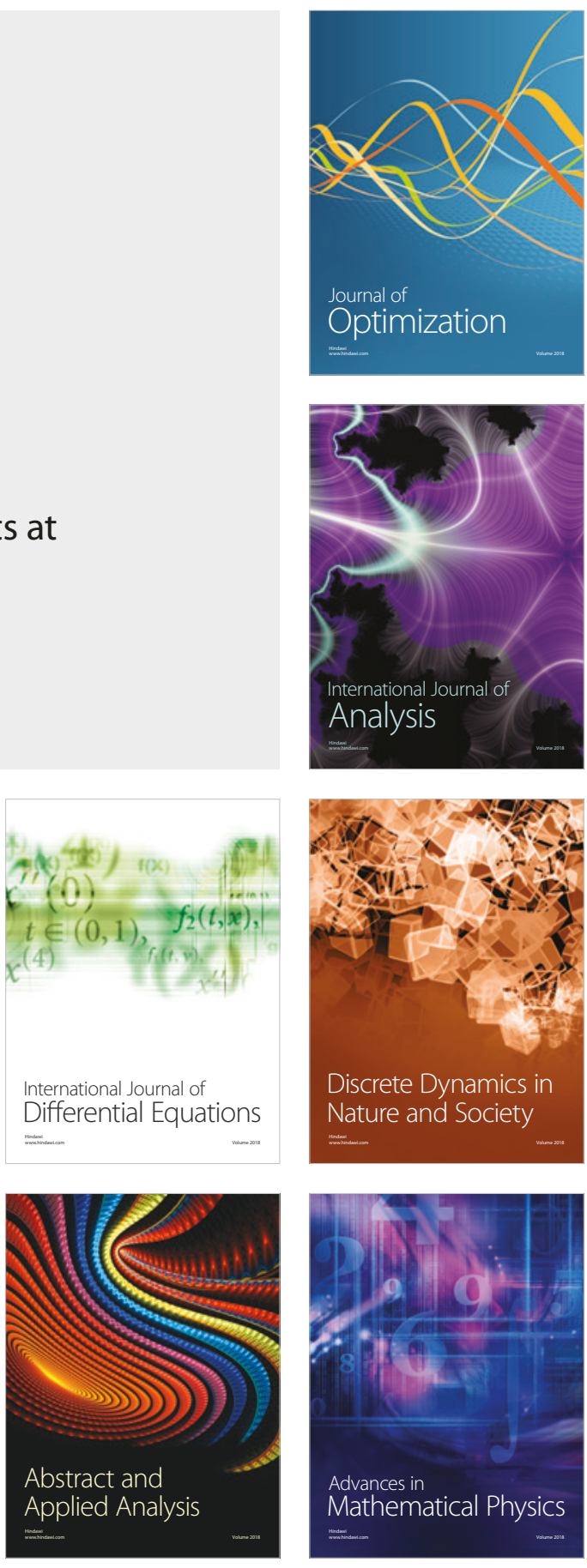